\title{
Induced polarization response of porous media with metallic particles - Part 8: Influence of temperature and salinity
}

\author{
André Revil ${ }^{1}$, Antoine Coperey ${ }^{2}$, Deqiang $\mathrm{Mao}^{3}$, Feras Abdulsamad ${ }^{2}$, Ahmad Ghorbani $^{4}$, \\ Magali Rossi ${ }^{5}$, and Dominique Gasquet ${ }^{5}$
}

\begin{abstract}
We have investigated the influence of temperature and salinity upon the spectral induced polarization of 10 samples including rocks with their mineralization (galena, chalcopyrite) plus sand mixed with semiconductors such as magnetite grains, graphite, and pyrite cubes of two different sizes. Measurements are made in a temperature-controlled bath with a high-precision impedance meter and using $\mathrm{NaCl}$ solutions. We cover the temperature range $5^{\circ} \mathrm{C}-50^{\circ} \mathrm{C}$ and the frequency range $10^{-2} \mathrm{~Hz}$ to $45 \mathrm{kHz}$. For one large pyrite cube, we also investigated six salinities from 0.1 to $10 \mathrm{~S} \mathrm{~m}^{-1}$ (at $25^{\circ} \mathrm{C}, \mathrm{NaCl}$ ) and three salinities for graphite. The spectra are fitted with a Cole-Cole complex parametric conductivity model for which we provide a physical meaning to the four Cole-Cole parameters. As expected, the Cole-Cole exponent and the chargeability are independent of
\end{abstract}

the temperature and salinity. The instantaneous and steady state (direct current [DC]) conductivities depend on the salinity and temperature. This temperature dependence can be fitted with an Arrhenius law (combining the Stokes-Einstein and VogelFulcher-Tammann equations) with an activation energy in the range of $15 \pm 1 \mathrm{~kJ} \mathrm{Mol}^{-1}$. This activation energy is the same as for the bulk pore-water conductivity demonstrating the control by the background electrolyte of these quantities, as expected. The instantaneous and DC conductivities depend on the salinity in a predictable way. The Cole-Cole relaxation time decreases with the temperature and decreases with the salinity. This behavior can be modeled with an Arrhenius law with an apparent activation energy of $7 \pm 3 \mathrm{~kJ} \mathrm{~mol}^{-1}$. A finite-element model is used further to analyze the mechanisms of polarization, and it can reproduce the temperature and salinity dependencies observed in the laboratory.

\section{INTRODUCTION}

Induced polarization is a geophysical method characterizing the ability of porous media to reversibly store electrical charges (e.g., Schlumberger, 1920; Vacquier et al., 1957). For environmental applications of the technique, interested readers are directed to the review papers by Kemna et al. (2012) and Revil et al. (2012). An understanding of the induced polarization signature of disseminated metallic particles is required for ore prospecting (Wait, 1959; Zonge and Wynn, 1975; Ostrander and Zonge, 1978; Zonge and Hughes,
1981; Meju, 2002; Yoshioka and Zhdanov, 2005; Shen et al., 2007; Liu et al., 2017), the localization of anticlines hosting oil reservoirs due to the formation of pyrite halos above these oil traps (e.g., Veeken et al., 2009; Flekkøy, 2013), and the monitoring of bioremediation of contaminant plumes (e.g., Ntarlagiannis et al., 2005; Flores Orozco et al., 2011; Mewafy et al., 2013; Abdel Aal et al., 2014). For all of these applications, knowing how temperature and salinity affects complex conductivity spectra is important to better forward model the induced polarization response expected in the subsurface thanks to the models developed in this series of papers

Manuscript received by the Editor 3 February 2018; revised manuscript received 24 May 2018; published ahead of production 25 August 2018; published online 9 November 2018 .

${ }^{1}$ Corresponding author. Université Grenoble Alpes, Université Savoie Mont Blanc, CNRS, IRD, IFSTTAR, ISTerre, 38000 Grenoble, France. E-mail: andre .revil@univ-smb.fr.

${ }^{2}$ Université Grenoble Alpes, Université Savoie Mont Blanc, CNRS, IRD, IFSTTAR, ISTerre, 38000 Grenoble, France. E-mail: andre.revil@univ-smb.fr; antoine.coperey@gmail.com; feras.abdulsamad@upmc.fr.

${ }^{3}$ Shandong University, School of Civil Engineering, 17922 Jingshi Road, Jinan 250061, China. E-mail: maodeqiang@sdu.edu.cn.

${ }^{4}$ Yazd University, Department of Mining and Metallurgical Engineering, Yazd, Iran. E-mail: aghorbani@yazd.ac.ir.

${ }^{5}$ Université de Savoie Mont-Blanc, Laboratoire EDYTEM-UMR 5204 du CNRS-Campus Scientifique, F-73376 Le Bourget-du-Lac, France. E-mail: magali .rossi@univ-smb.fr; dominique.gasquet@univ-savoie.fr.

(C) 2018 Society of Exploration Geophysicists. All rights reserved. 
Table 1. Properties of the clean sand and minerals used in the experiments. The formation factor of the clean sand was determined using in-phase conductivity data at $1 \mathrm{~Hz}$ and two pore-water salinities $\left(1^{-2}\right.$ and $\left.10^{-1} \mathrm{M}, \mathrm{NaCl}\right)$. The porosity of the sand and the density of the pyrite were obtained from weight and volume measurements. The P1 pyrite corresponds to a single large cubic grain, whereas the P2 pyrite corresponds to a single small cubic grain (Figure 1). For the magnetite sample M1, we use a total of $11 \mathrm{~cm}$ size grains. The density of sample G2 can be compared with the density of pure lead $\left(11,340 \mathrm{~kg} \mathrm{~m}^{-3}\right)$.

\begin{tabular}{lcc}
\hline & Symbol & Value \\
\hline Parameter & $F_{S d}$ & $3.7 \pm 0.2$ \\
Formation factor clean sand & $d$ & $200 \pm 20 \mu \mathrm{m}$ \\
Pean grain diameter sand & $\varphi_{S d}$ & $0.40 \pm 0.03$ \\
Grain diameter pyrite (P1) & $a$ & $2.9 \pm 0.2 \mathrm{~cm}$ \\
Grain diameter pyrite (P2) & $a$ & $1.4 \pm 0.2 \mathrm{~cm}$ \\
Grain diameter graphite (Gr2) & $a$ & $(9 \pm 0.2) \times(3 \pm 0.2) \mathrm{cm}$ \\
Grain diameter galena (G2) & $a$ & $(5.4 \pm 0.2) \times(2.8 \pm 0.2) \mathrm{cm}$ \\
Grain diameter magnetite (M1) & $a$ & $1 \pm 0.2 \mathrm{~cm}$ \\
Mass density pyrite (P1) & $\rho_{m}$ & $4400 \mathrm{kgm}^{-3}$ \\
Mass density pyrite (P2) & $\rho_{m}$ & $4700 \mathrm{kgm}^{-3}$ \\
Mass density magnetite (M1) & $\rho_{m}$ & $5200 \mathrm{kgm}^{-3}$ \\
Mass density graphite (Gr2) & $\rho_{m}$ & $2110 \mathrm{kgm}^{-3}$ \\
Mass density galena (G2) & $\rho_{m}$ & $6860 \mathrm{kgm}^{-3}$ \\
\hline
\end{tabular}

(Revil et al., 2015a, 2015b, 2017a, 2017b; Mao and Revil, 2016; Mao et al., 2016).

An additional motivation of the present study is to test existing mechanistic models by looking at the mechanisms of induced polarization of disseminated metallic particles in a background electrolyte or electrolyte-saturated porous matrix. We want to determine if the dominant polarization is ionic in nature (i.e., associated with the relaxation of the field-induced electrical diffuse layers [DLs]) or associated with the electrodiffusion of electrons and p-holes inside the semiconductors themselves (for a description of such transport mechanisms in semiconductors, see Rajeshwar, 2007), or a composite of both. Indeed, the temperature dependence of the mobility of the charge carriers belonging to the metallic particles themselves should be pretty different from the temperature dependence of the mobility of the ions in the pore water. For ionic processes, the activation energy controlling the mobility of the charge carriers (and therefore the diffusion coefficient) is the same as the activation energy controlling the dependence of the dynamic viscosity of the pore water with temperature (approximately $15 \pm 1 \mathrm{~kJ} \mathrm{Mol}^{-1}$, i.e., approximately $0.16 \mathrm{eV}$ per molecule, see Viktor and Hazra, 2002; Toumi et al., 2009).

Surprisingly, as far as we know, the temperature dependence of complex conductivity spectra of porous materials with disseminated ores has never been explored. Several works have focused on the temperature dependence of the complex conductivity of porous materials with no metallic particles (Vinegar and Waxman, 1984; Tong and Tao, 2007; Binley et al., 2010; Zisser et al., 2010; Bairlein et al., 2016). This dependence is fairly well-explained by the current model of the dynamic Stern layer model with a mobility of the charge carriers depending on temperature according to either a linear or an Arrhenius law (e.g., Zisser et al., 2010; Revil et al.,

Table 2. List of the 17 experiments performed in the present study. We use $\mathrm{NaCl}$ solutions, and $\sigma_{w}$ denotes the conductivity of the pore water at $25^{\circ} \mathrm{C}$. The background is made of sand. The quantity $\varphi_{m}$ denotes the volume fraction of metallic particles in the mixture. $S$ denotes the pure sand sample. FD and TD stand for the frequency-domain and time-domain measurements, respectively. We used an applied voltage of $0.2 \mathrm{~V}$ by default and $3 \mathrm{~V}$ for frequency-domain and time-domain measurements, respectively. The conductivity of the pore water is given at $25^{\circ} \mathrm{C}$.

\begin{tabular}{|c|c|c|c|c|c|}
\hline Experiment & Material & $\varphi_{m}(-)$ & Type & Temperature range & $\sigma_{w}\left(\mathrm{~S} \mathrm{~m}^{-1}\right)$ \\
\hline 2 & P1-Pyrite & 0.26 & FD & $5^{\circ} \mathrm{C}-50^{\circ} \mathrm{C}$ & 1.0 \\
\hline 4 & P1-Pyrite & 0.26 & FD & $5^{\circ} \mathrm{C}-50^{\circ} \mathrm{C}$ & 10 \\
\hline 5 & P2-Pyrite & 0.04 & FD & $5^{\circ} \mathrm{C}-50^{\circ} \mathrm{C}$ & 0.1 \\
\hline 8 & S-Sand & 0.00 & FD & $5^{\circ} \mathrm{C}-50^{\circ} \mathrm{C}$ & $0.1-1.0$ \\
\hline 9 & G1-Galena (lead) & - & FD & $5^{\circ} \mathrm{C}-50^{\circ} \mathrm{C}$ & 0.1 \\
\hline 10 & G2-Galena (lead) & - & FD & $5^{\circ} \mathrm{C}-50^{\circ} \mathrm{C}$ & 0.1 \\
\hline 11 & C2-Chacopyrite & - & FD & $5^{\circ} \mathrm{C}-50^{\circ} \mathrm{C}$ & 0.1 \\
\hline 15 & P1-Pyrite & 0.26 & FD & $5^{\circ} \mathrm{C}-50^{\circ} \mathrm{C}$ & 2 \\
\hline 16 & Gr2-Graphite & 0.17 & FD & $5^{\circ} \mathrm{C}-50^{\circ} \mathrm{C}$ & 1.0 \\
\hline 17 & Gr2-Graphite & 0.17 & FD & $5^{\circ} \mathrm{C}-50^{\circ} \mathrm{C}$ & 5.0 \\
\hline
\end{tabular}


a)

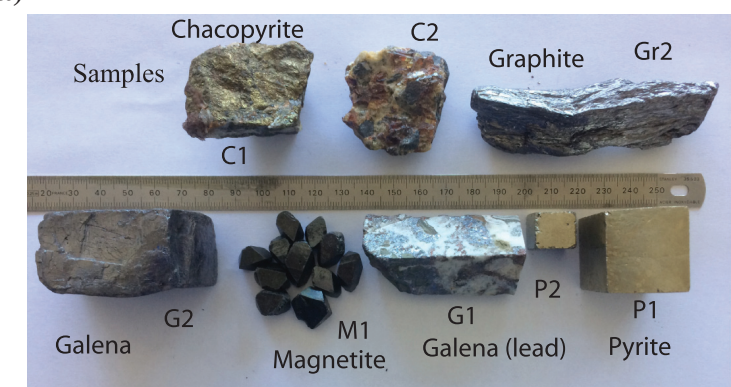

b)

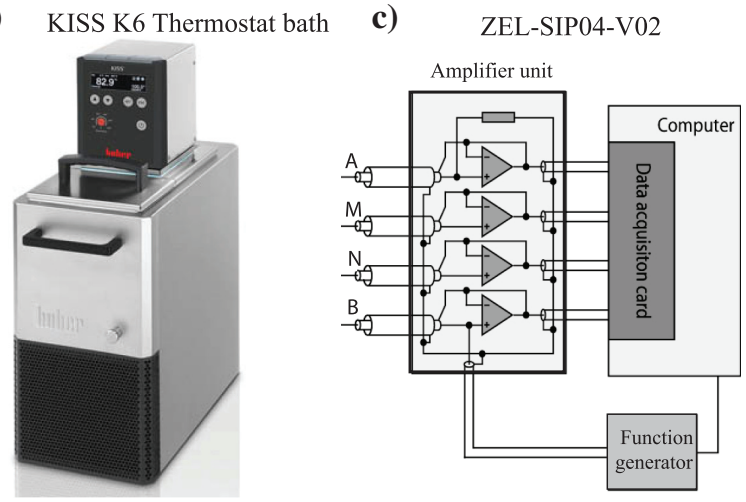

Figure 1. Core samples and equipment. (a) Minerals and core samples used for the experiments. P1 is a pyrite cube of $3 \mathrm{~cm}$ in size. (b) Thermostat bath. (c) High-precision impedance meter with A and $\mathrm{B}$ being the current electrodes and $\mathrm{M}$ and $\mathrm{N}$ being the voltage electrodes.

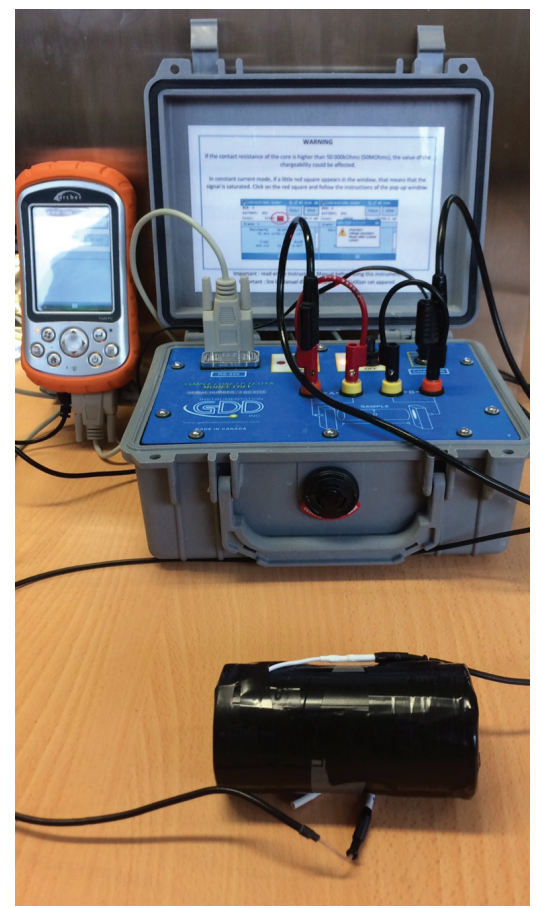

Figure 2. SCIP from GDD Inc. used for the time-domain measurements with four electrodes (A, B, M, and N). We used the following set of time-on: 1.0, 2.0, and $4.0 \mathrm{~s}$, and we stack four times. We use 20 windows respecting a Cole-Cole distribution. We use an imposed voltage of $3 \mathrm{~V}$. 2017c). In addition, we are interested to test the salinity dependence of the Cole-Cole parameters used to model the complex conductivity response of sulfide-bearing materials in freshwater or saline environments. Note that previous works have noticed a perhaps unexpected dependence of the Cole-Cole relaxation time of dispersed metallic grains with the salinity of the pore water (Slater et al., 2005, their Figure 5a; Gurin et al., 2015; Hupfer et al., 2016, their Figure 11c). This dependence is however not explained by the classical theories (redox-controlled polarization, Wong, 1979; or electrical field-induced DL polarization, Chu and Bazant, 2006).

In this paper, we selected 10 samples including semiconductors (such as pyrite and magnetite), metals (such as lead), and a semimetal (graphite) mixed with a well-calibrated sand. The complex conductivity measurements (17 experiments in total) were performed in a temperature-controlled bath in which the imposed temperature is highly controlled (approximately $0.1^{\circ} \mathrm{C}$ ) and can be maintained for a long duration (days if needed) to be sure that a complete thermal equilibrium state has been reached. The complex conductivity spectra were fitted with a Cole-Cole parametric a)

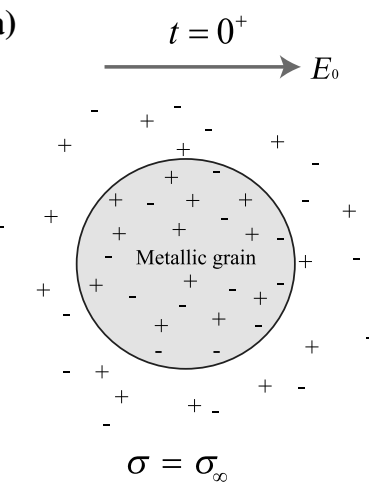

c)

$$
\tau_{i} \gg t>\tau_{e}
$$

No external field

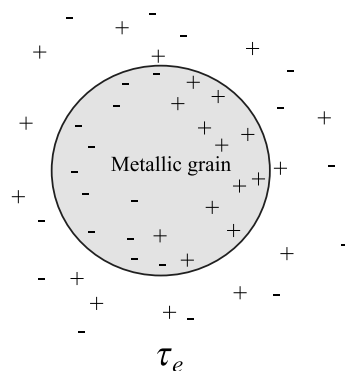

b)

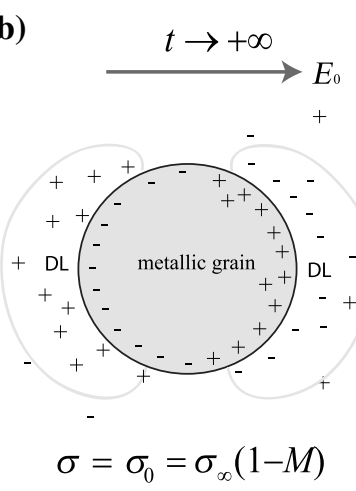

d)

$$
t>\tau_{e}, \tau_{i}
$$

No external field

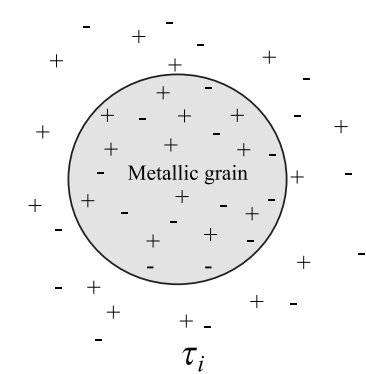

Figure 3. Polarization of a metallic particle. (a) Just after the application of the external electrical field $\mathbf{E}_{0}$, all the charge carriers inside (electrons and p-holes) and outside (cations and anions) the metallic particle are mobile providing the instantaneous conductivity $\sigma_{\infty}$. The metallic particle appears highly conductive. (b) For a very long application of the external electrical field, the metallic particle is entirely polarized and appears as an insulator (i.e., the charge carriers are not mobile anymore). The generated surface charges are responsible for the formation of field-induced electrical DLs in the vicinity of the metallic particle. This situation defined the DC conductivity of the material $\sigma_{0}$, which is necessarily smaller than the instantaneous conductivity. (c) Relaxation of the external charges in the electrolyte with a relaxation time of $\tau_{e}$. (d) Relaxation of the internal charges in the metallic particle with a relaxation time of $\tau_{i}$. 
model. We also investigated the thermal dependence of (1) a pyrite cube with a background sand at six different salinities and (2) a graphite porous bar at three salinities to see how the change of the conductivity of the background material around the metallic particles affects the Cole-Cole parameters.

\section{MATERIALS AND METHODS}

\section{Materials}

We use a new collection of 10 samples described in Table 1 (see also Figure 1), and we investigated six salinities for one of the core sample (a large pyrite cube, approximately $3 \mathrm{~cm}$, called P1 below) and three salinities for a second graphite sample. The minerals and rocks include chalcopyrite, graphite, galena, magnetite, and two pyrite cubes. Seventeen experiments were performed mostly in the frequency domain with also a few experiments performed in the time domain (see details in Table 2 and Figure 2). For the mixes between the metallic particles and the sand, we use a pure silica sand, whose properties are reported in Table 1. The properties and characteristics of the minerals used in our experiments are also reported in Table 1.

\section{Frequency-domain measurements}

The goal of the experiments will be to check the conceptual model sketched in Figure 3 through temperature-controlled experiments, some of them being performed at different salinities. We will come back later on the polarization mechanisms involved in Figure 3 all along this paper. The temperature-controlled bath and the ZEL-SIP04-V02 impedance meter (frequency-domain measurements) are used in concert (see Figure $1 \mathrm{~b}$ and $1 \mathrm{c}$, respectively). The ZEL-SIP04-V02 impedance meter was developed by Egon Zimmermann at the Central Laboratory for Electronics, ZEL, the Forschungszentrum Julich (see Zimmermann et al., 2007). Benchmark tests can be found, for instance, in Revil and Skold (2011) and

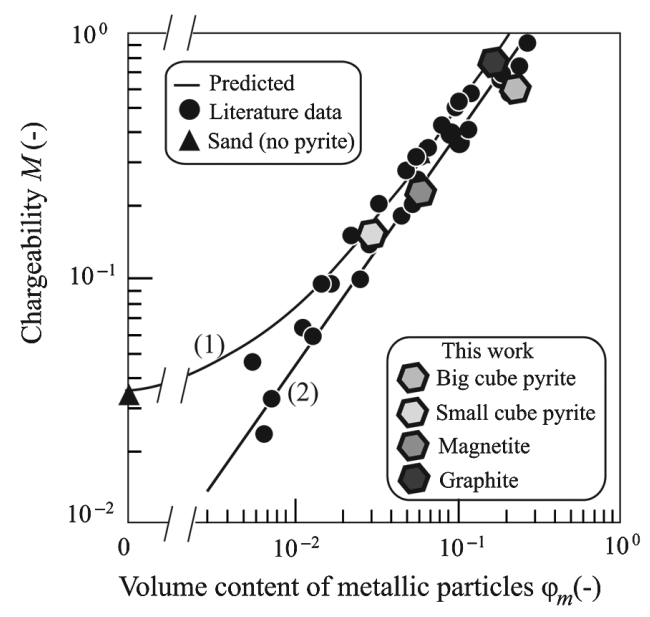

Figure 4. Comparison between the chargeability determined at $25^{\circ} \mathrm{C}$ and the volume fraction of the metallic particle mixed with sand. The two curves (1, with sand background) and (2, without background) correspond to equation 3 with and without background polarization, respectively. The new data correspond to the average for experiments 1-7. The two pyrite cubes correspond to P1 and P2 (see Tables 1 and 2). References from the literature: Pelton et al. (1978), Mahan et al. (1986), Phillips (2010), and Gurin et al. (2015).
Woodruff et al. (2014) among others and will not be repeated here (for experiments performed with ore materials, see also Revil et al., 2017a, 2017b). The typical accuracy of this high-precision impedance meter is $0.1 \mathrm{mrad}$ between $1 \mathrm{mHz}$ and $1 \mathrm{kHz}$ and $0.4 \mathrm{mrad}$ between 1 and $45 \mathrm{kHz}$. We used an applied voltage of \pm 0.2 or $\pm 1 \mathrm{~V}$ for our experiments (higher voltages can yield nonlinear effects and, while interesting, were avoided in this study to stay in the linear regime; for a discussion of the nonlinear regime, see Chu and Bazant, 2006).

The methodology used for our experiments is essentially the same as in Revil et al. (2017a) and will not be repeated here. The sample holder (described below) is put in a heat-resistant insulating bag immersed in a thermostat bath (KISS K6 from Huber, dimensions $210 \times 400 \times 546 \mathrm{~mm}$, heating capacity $2 \mathrm{~kW}$, bath volume 4.5 L; see Figure $1 \mathrm{~b}$ ). The temperature of this bath is controlled with a precision of $0.1^{\circ} \mathrm{C}$. Glycol is used as a heat-carrying fluid. At each temperature, we allow enough time ( $>1 \mathrm{~h}$, sometimes more) for the system to stabilize in temperature. An additional check of the stabilization was done through repeated in-phase conductivity measurements until a plateau was reached.
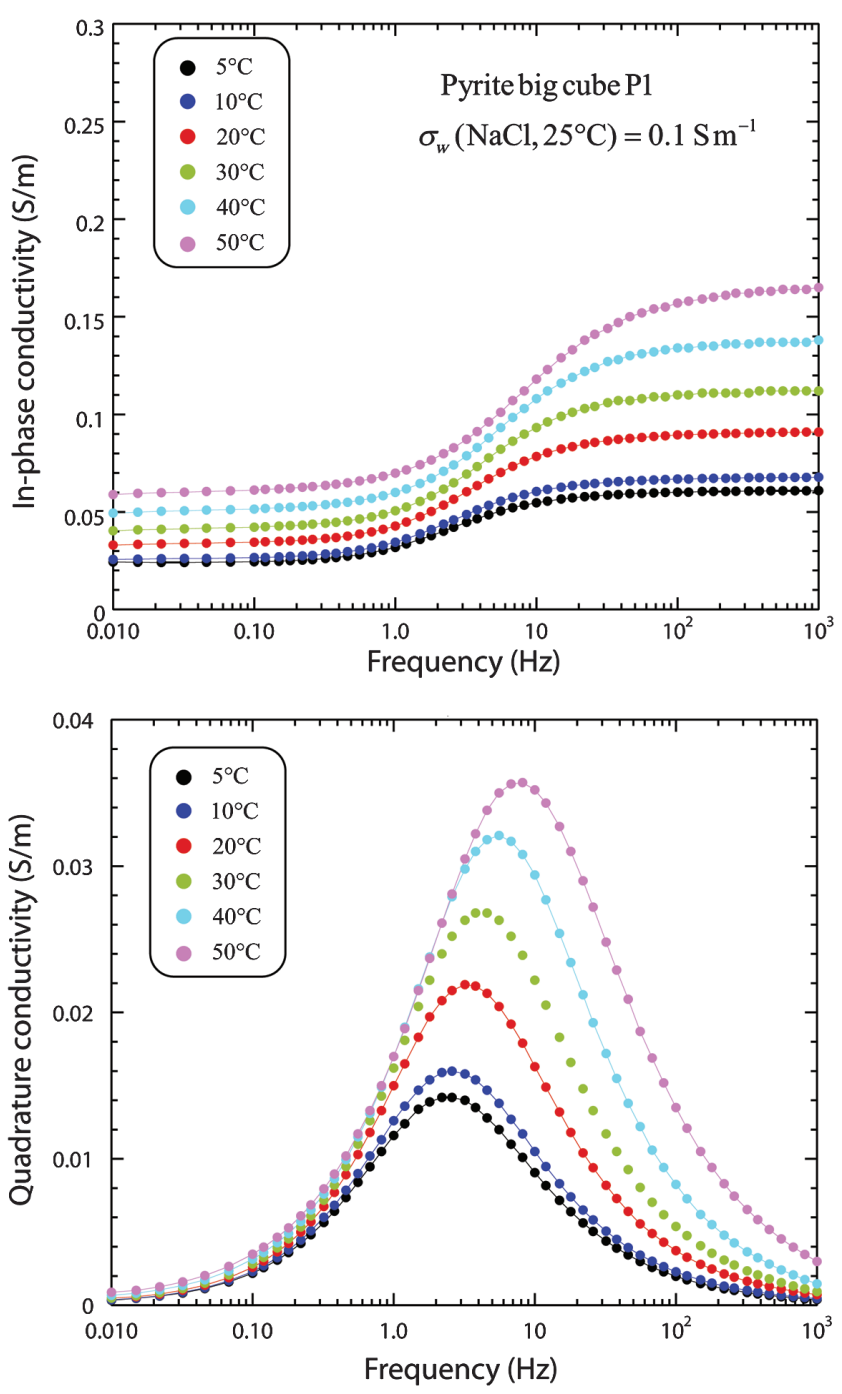

Figure 5. Experiment 1 (low salinity $0.1 \mathrm{~S} \mathrm{~m}^{-1} \mathrm{NaCl}$ at $25^{\circ} \mathrm{C}$, big pyrite cube P1; see Table 2 ). The plain lines are guides for the eyes. 
We use a cylindrical sample holder with two current electrodes A and B placed at the end faces of the sample. This configuration is used to impose a uniform current through the sample. The two potential electrodes $\mathrm{M}$ and $\mathrm{N}$ are in contact with the core sample on the side of the sample holder. Because of the small size of the contact area between the voltage electrodes $\mathrm{M}$ and $\mathrm{N}$ and the core sample, they are considered as point electrodes. The conversion between the measured impedance into the complex conductivity was done by using a geometric factor $K$ depending on the geometry of the electrodes $(K=L / A$ in the present case, where $A$ is the surface area of a cross section of the cylindrical core sample and $L$ is the distance between the voltage electrodes $\mathrm{M}$ and $\mathrm{N}$ ).

The core samples were prepared as follows. An electrolyte was prepared at the desired salinity using dehydrated $\mathrm{NaCl}$ salt (with a high purity level) and deionized water. Then, the brine was degassed for $30 \mathrm{~min}$ with a very low-pressure pump reaching the vapor pressure (approximately $15 \mathrm{~Pa}$ ). The brine is used to saturate the porous material. The complex conductivity spectra were taken $24 \mathrm{~h}$ after the preparation of the mixture in the sample holder following the same rules discussed in detail in Revil et al. (2017a, 2017b). The frequency-domain measurements were performed from $10 \mathrm{mHz}$ to $45 \mathrm{kHz}$. At each frequency, the standard deviations are determined using two cycles. The temperature range investigated in the present study is $5^{\circ} \mathrm{C}-50^{\circ} \mathrm{C}$ (Table 2). The classical dependence between the chargeability and the volume content of metal shown in Figure 4 should be preserved at any temperature. Typical frequency-domain spectra at different temperatures and salinities are shown in Figures 5, 6, 7, 8, and 9 .

\section{Time-domain measurements}

We also performed induced polarization measurement in the time domain using the sample core I.P. tester (SCIP) from GDD Inc. (Figure 2). For each measurement, we recorded the resistance (in $\mathrm{ohm}$ ), the total chargeability (in $\mathrm{mV} / \mathrm{V}$ ), and the chargeability for each window. We used three different time-on: 1.0, 2.0, and $4.0 \mathrm{~s}$
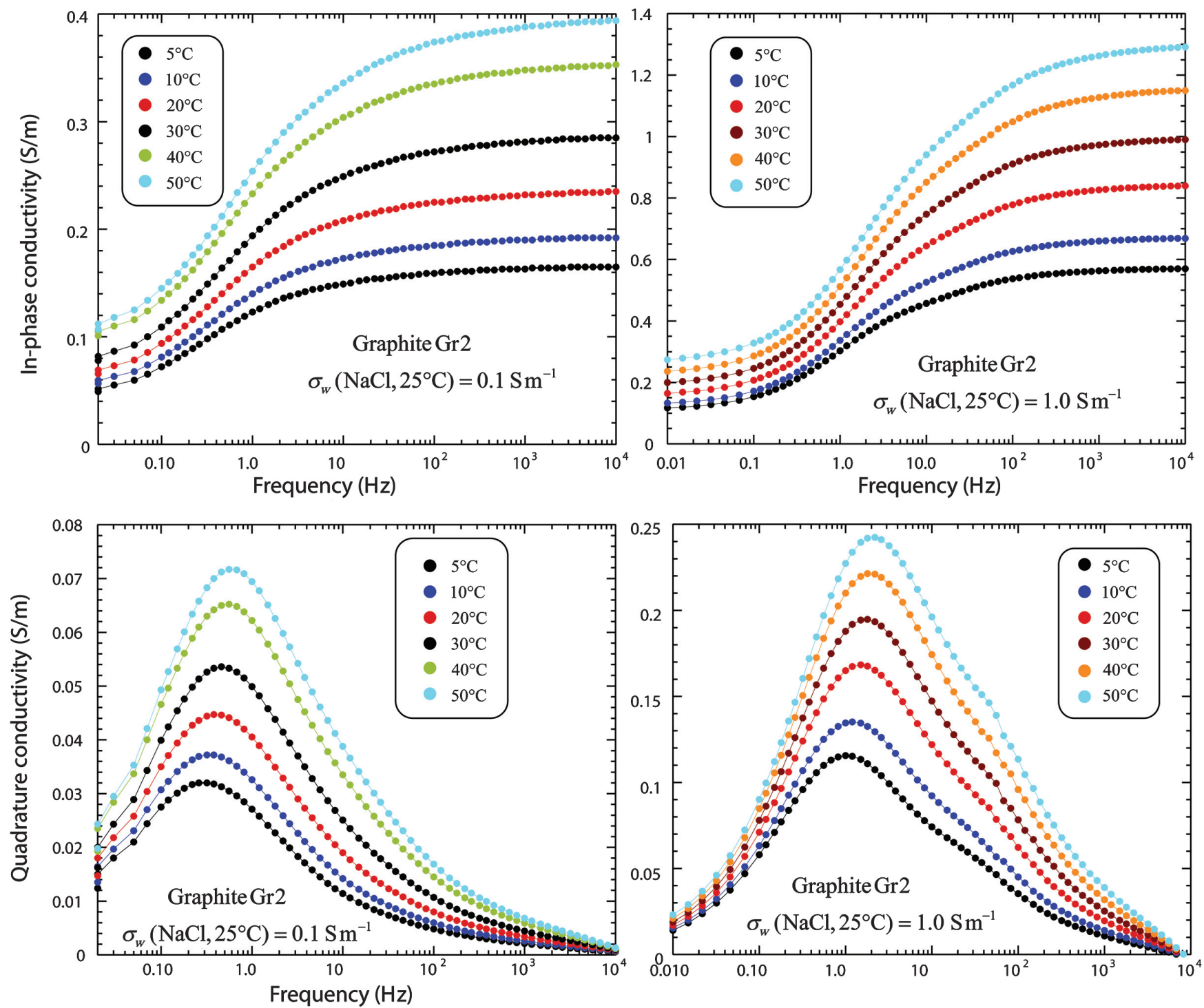

Figure 6. Experiments 7 (at $0.1 \mathrm{~S} \mathrm{~m}^{-1} \mathrm{NaCl}$ at $25^{\circ} \mathrm{C}$, graphite $\mathrm{Gr} 2$, see Table 2) and 16 (at $1.0 \mathrm{~S} \mathrm{~m}^{-1} \mathrm{NaCl}$ at $25^{\circ} \mathrm{C}$, graphite $\mathrm{Gr} 2$; see Table 2). The plain lines are guides for the eyes. 
and stacks is equal to four. Usually, the number of windows is 20 respecting a Cole-Cole distribution. All measurements were done with an injection current of $3 \mathrm{~V}$. We use the pyrite cube P1 (brine solution at $\left.1.0 \mathrm{~S} \mathrm{~m}^{-1}, \mathrm{NaCl}, 25^{\circ} \mathrm{C}\right)$. The measurements were done at temperatures $\left(5^{\circ} \mathrm{C}, 10^{\circ} \mathrm{C}, 20^{\circ} \mathrm{C}, 30^{\circ} \mathrm{C}, 40^{\circ} \mathrm{C}\right.$, and $\left.50^{\circ} \mathrm{C}\right)$. The current electrodes are located at the end faces (electrodes $\mathrm{A}$ and $\mathrm{B}$ ) of the core sample, whereas the potential electrodes $\mathrm{M}$ and $\mathrm{N}$ are located on the surface of the core sample at some distance from each other in the direction of the imposed electrical field.

\section{COLE-COLE PARAMETERS}

\section{A Cole-Cole complex conductivity model}

We write the complex conductivity as $\sigma^{*}=\sigma^{\prime}+i \sigma^{\prime \prime}$, where $\sigma^{\prime}$ and $\sigma^{\prime \prime}$ in $\left(\mathrm{S} \mathrm{m}^{-1}\right)$ denote the in-phase and quadrature components. We consider only the linear response of a system composed of disseminated metallic grains in an ionic background such as a watersaturated sand (for the modeling of nonlinear effects, see Chu and Bazant, 2006; for their measurements, see Olhoeft, 1985). In this work, we assume that the complex conductivity of a porous material with disseminated semiconductors, such as pyrite (assuming a unimodal grain size distribution of the metallic particles) is given by a
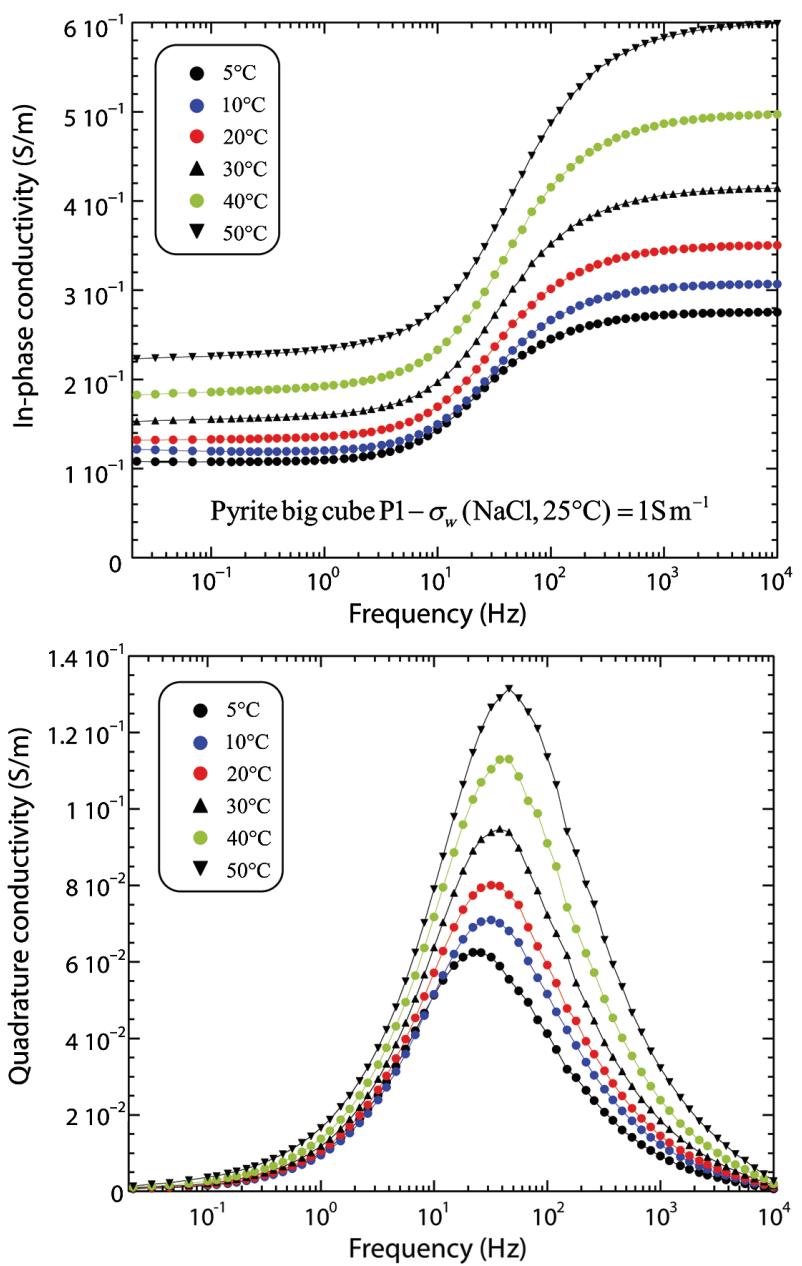

Figure 7. Experiment 2 (big pyrite cube $\mathrm{P} 1, \mathrm{NaCl}, 1.0 \mathrm{~S} \mathrm{~m}^{-1}$ at $25^{\circ} \mathrm{C}$ ). The plain lines are guides for the eyes.
Cole-Cole parametric model (see Revil et al., 2017a, their equation 11)

$$
\sigma^{*}=\sigma_{\infty}\left(1-\frac{M}{1+\left(i \omega \tau_{0}\right)^{c}}\right),
$$

where $c$ (dimensionless) denotes the Cole-Cole exponent, which describes the broadness of the relaxation time distribution, $\tau_{0}$ denotes the Cole-Cole relaxation time (in s), $\sigma_{\infty}$ denotes the instantaneous conductivity, and $M=\left(\sigma_{\infty}-\sigma_{0}\right) / \sigma_{\infty}$ denotes the chargeability (dimensionless; see Figure 4$)$. The instantaneous conductivity $\sigma_{0}\left(<\sigma_{\infty}\right)$ denotes the conductivity when all the charge carriers are mobile (Figure 3a). The DC conductivity $\sigma_{0}\left(<\sigma_{\infty}\right)$ denotes the conductivity in steady-state conditions of the application of an external electrical field (Figure 3b). The DC conductivity is smaller than the instantaneous conductivity because some electrical charges have lost their mobility and are now blocked by the process of polarization in and around the metallic particles (Figure $3 \mathrm{a}$ and $3 \mathrm{~b}$ ). There are several ways to physically derived a Cole-Cole model for the complex conductivity of dispersed metallic particles (see, for instance, Flekkøy, 2013).
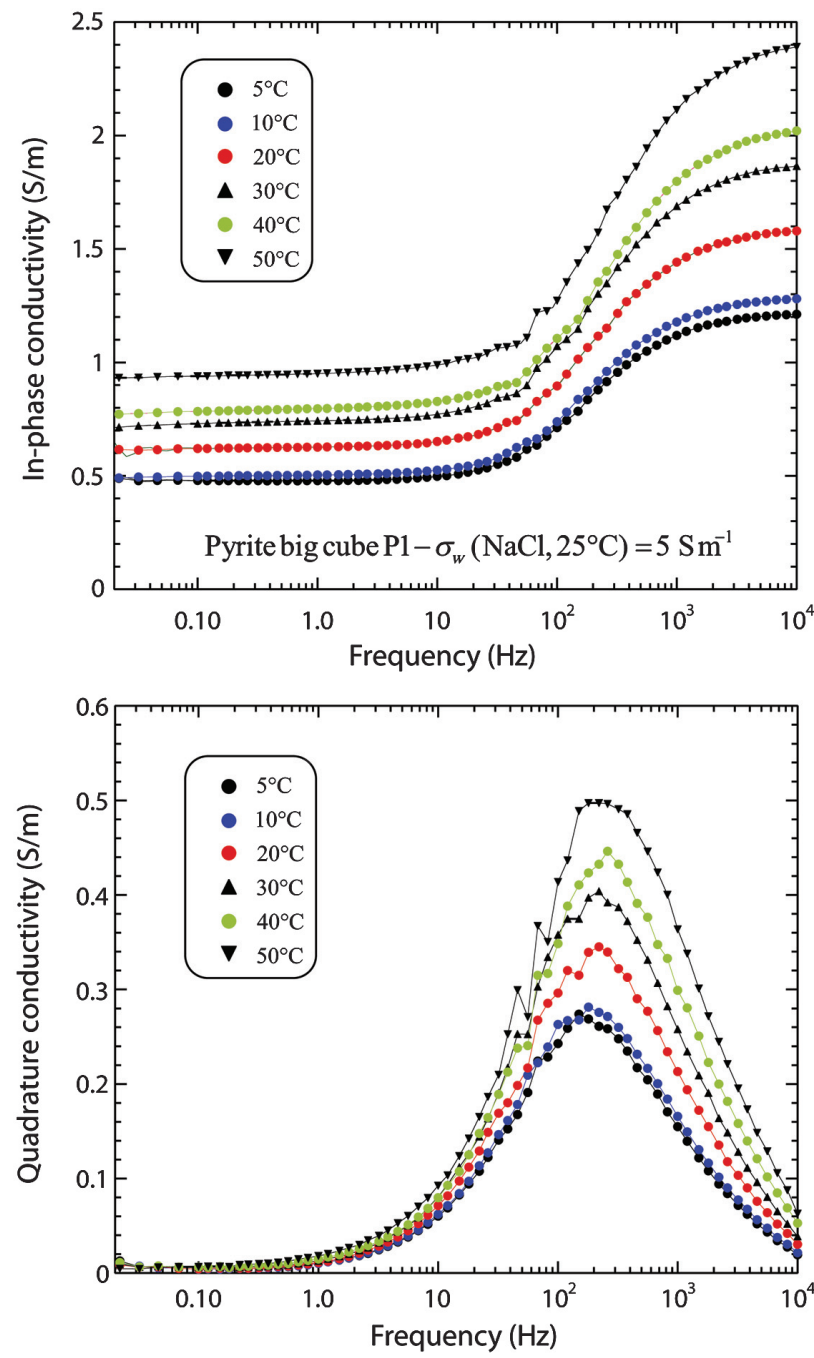

Figure 8. Experiment $4\left(\mathrm{P} 1, \mathrm{NaCl}, 5.0 \mathrm{~S} \mathrm{~m}^{-1}\right.$ at $\left.25^{\circ} \mathrm{C}\right)$. The plain lines are guides for the eyes. 
The Cole-Cole exponent $c$ denotes the broadness of the distribution of relaxation times. Because all of the relaxation times are supposed to have the same temperature dependence, $c$ is expected to be temperature independent. The chargeability $M$ entering equation 1 is directly related to the (dimensionless) volume content of metallic particles $\varphi_{m}$ (Revil et al., 2015a; see their equations 69 and 70)

$$
\begin{gathered}
M \approx 1-\left(1-\frac{9}{2} \varphi_{m}\right)\left(1-M_{b}\right), \\
M \approx \frac{9}{2} \varphi_{m}+M_{b},
\end{gathered}
$$

where $M_{b}$ (dimensionless) denotes the chargeability of the background material. Figure 4 shows how equation 3 agrees well with some available experimental data, including some data from the cur-
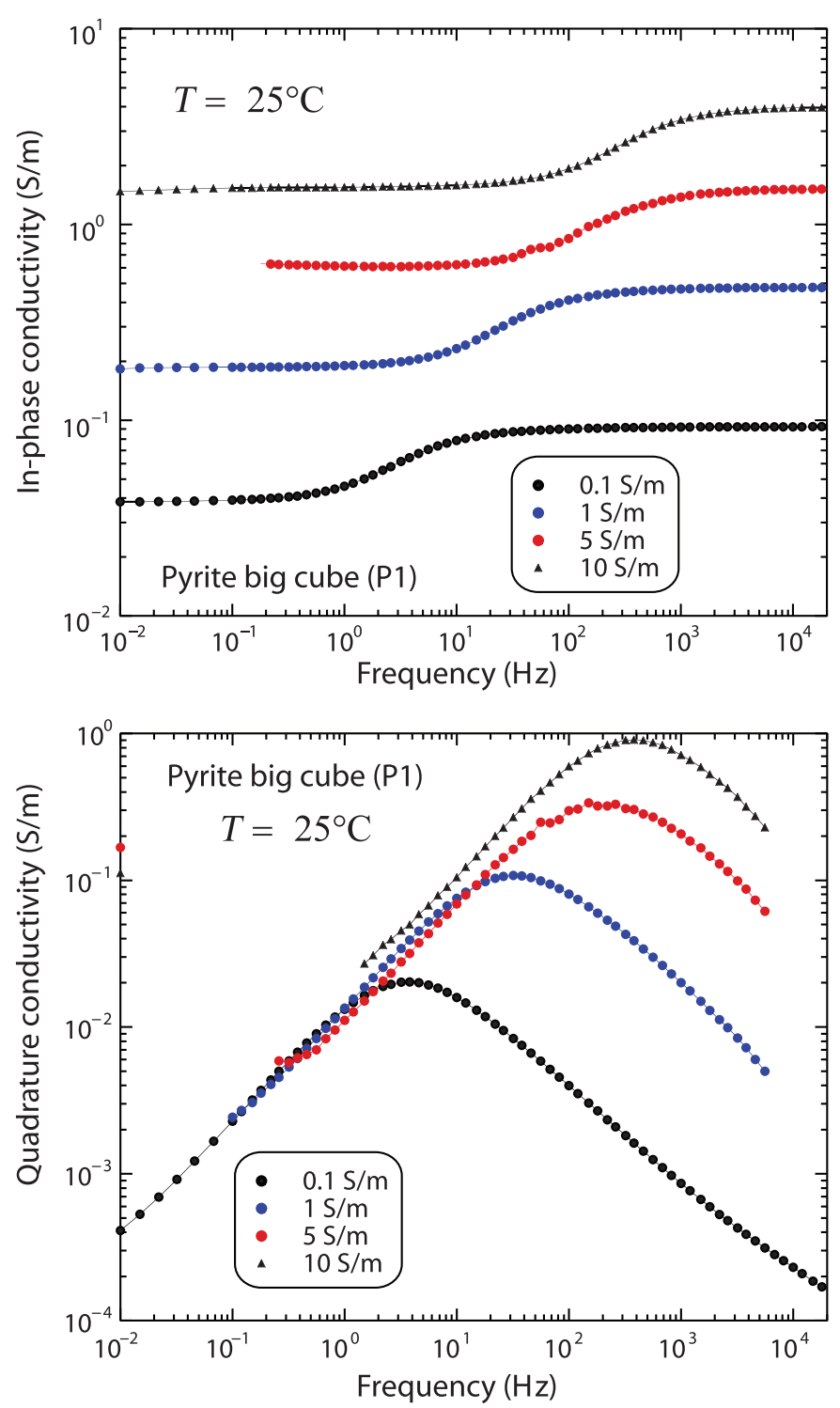

rent study. We have already shown that $\partial M_{b} / \partial T=0$ (Revil et al., $2017 \mathrm{c}$ ); i.e., the chargeability of the background material (here the sand) does not depend on the temperature. Therefore, it is expected that the chargeability of the mixture of the background material with the metallic grains would be independent of the temperature; i.e., $\partial M / \partial T=0$.

The instantaneous and the steady state conductivities are related to the instantaneous $\left(\sigma_{b}^{\infty}\right)$ and steady state $\left(\sigma_{b}^{0}\right)$ conductivities of the background material (subscript $b$ ) according to (Revil et al., 2015a)

$$
\sigma_{\infty} \approx \sigma_{b}^{\infty}\left(1+3 \varphi_{m}+\cdots\right)
$$

$$
\sigma_{0} \approx \sigma_{b}^{0}\left(1-\frac{3}{2} \varphi_{m}+\cdots\right)
$$
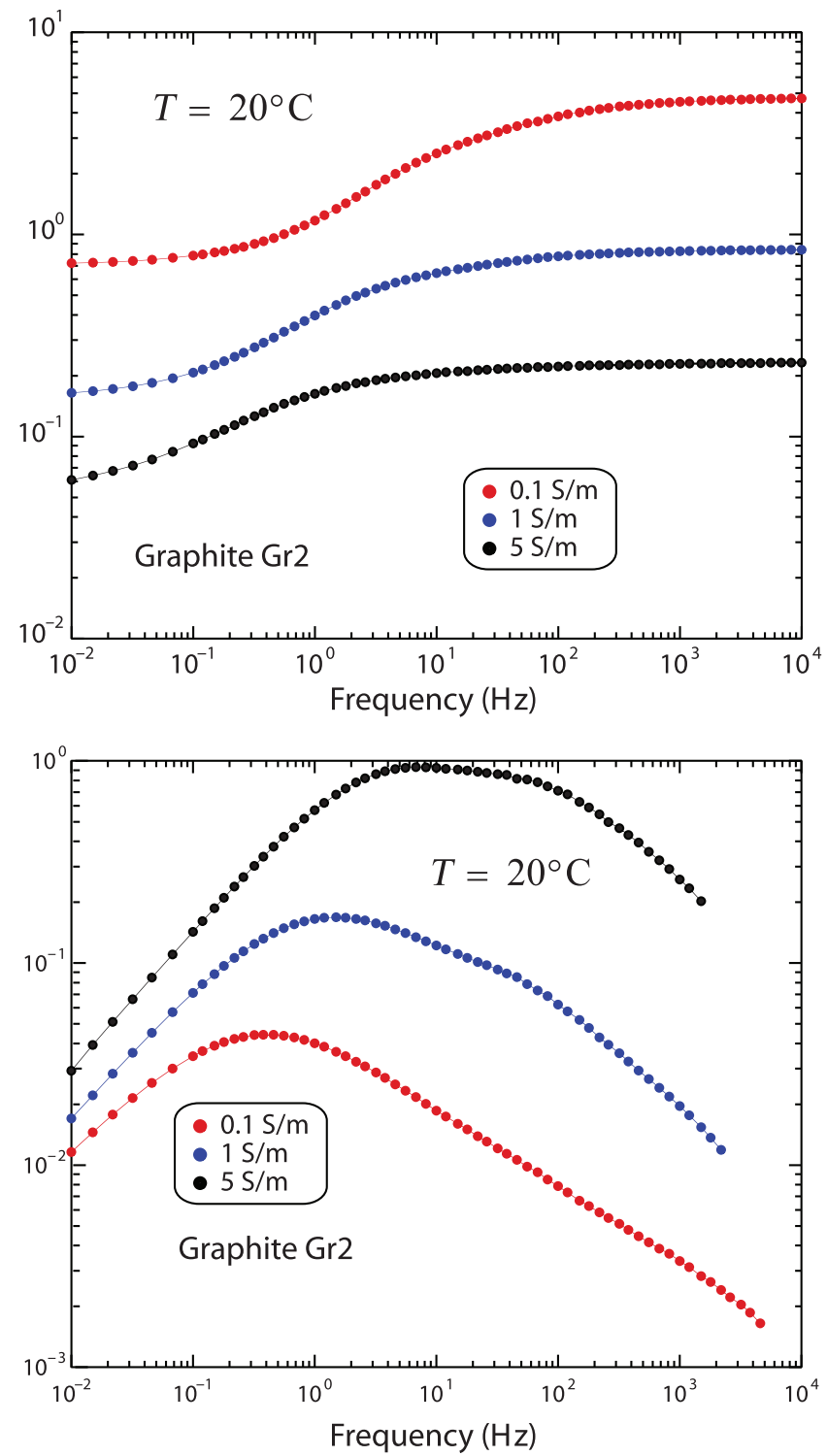

Figure 9. Experiments 1 to 4 (pyrite $\mathrm{P} 1$ at $0.1,1.0,5.0$, and $10 \mathrm{~S} \mathrm{~m}^{-1} \mathrm{NaCl}$ at $25^{\circ} \mathrm{C}$, big pyrite cube $\mathrm{P} 1$; see Table 2) and experiments 7,16 , and 17 (graphite $\mathrm{Gr} 2$ at $0.1,1.0$, and $5.0, \mathrm{~S} \mathrm{~m}^{-1} \mathrm{NaCl}$ at $25^{\circ} \mathrm{C}$; see Table 2). 
Note that if the background if made of sand, the conductivity depends very weakly on the frequency and therefore, $\sigma_{b}^{0} \approx \sigma_{b}^{\infty}$. We will note $\sigma_{b}$ the conductivity of the background. It follows that the temperature dependence of the instantaneous and steady-state conductivities of the composite (metallic particles and background) is equal to the temperature dependence of the instantaneous and steady-state conductivities of the background, which are known (see Revil et al., 2017c) and are given by

$$
\sigma_{b}(T)=\sigma_{b}\left(T_{0}\right)\left(1+\alpha_{w}\left(T-T_{0}\right)\right),
$$

where $T$ and $T_{0}$ are expressed in degree Celsius $\left({ }^{\circ} \mathrm{C}\right)$ and where the reference temperature is $T_{0}=25^{\circ} \mathrm{C}, \alpha_{w}$ is the sensitivity coefficient for the temperature dependence of the charge carriers of the background material. In the temperature range $0^{\circ} \mathrm{C}-50^{\circ} \mathrm{C}$ for which we can consider linear temperature variations (Campbell et al., 1948; Vinegar and Waxman, 1984), this temperature dependence would be similar to the temperature dependence of the pore-water conductivity $\sigma_{w}(T)$ given by
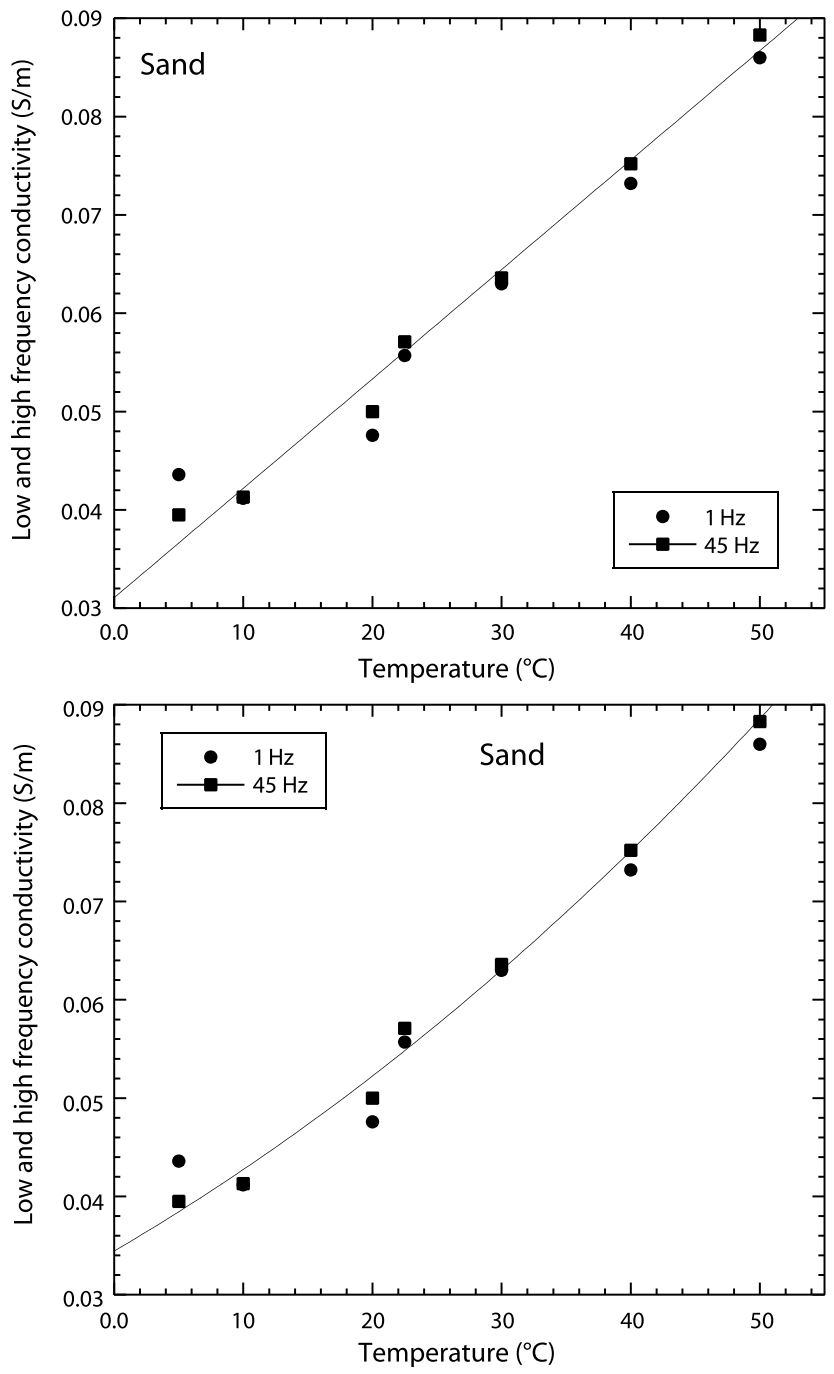

Figure 10. Dependence of the low- and high-frequency conductivities with temperature for the clean sand alone at $0.1 \mathrm{~S} \mathrm{~m}^{-1}(\mathrm{NaCl}$ at $25^{\circ} \mathrm{C}$ ) (experiment 8).

$$
\sigma_{w}(T)=\sigma_{w}\left(T_{0}\right)\left[1+\alpha_{w}\left(T-T_{0}\right)+\cdots\right] .
$$

For water, we have $\alpha_{w} \approx 0.020 \pm 0.002^{\circ} \mathrm{C}^{-1}$. For instance, considering experiment 8 , with just a clean sand, the temperature dependence of the conductivity is $\alpha_{w}=0.019 \pm 0.001^{\circ} \mathrm{C}^{-1}$ (see Figure 10).

We infer therefore that the instantaneous and DC conductivity of the mixture depend on temperature according to

$$
\sigma_{\infty}(T)=\sigma_{\infty}\left(T_{0}\right)\left(1+\alpha_{w}\left(T-T_{0}\right)\right)
$$

$$
\sigma_{0}(T)=\sigma_{0}\left(T_{0}\right)\left(1+\alpha_{w}\left(T-T_{0}\right)\right)
$$

respectively. Alternatively, we can also use an Arrhenius equation to fit the data using an activation energy $E_{a}^{w}$ (this choice is explained in more detail in Appendix A). The rationale behind the use of this equation is that the hydrated ions have to move through the water molecules, which are offering some viscous resistance to the movement of the ions by electromigration or diffusion. The temperature dependence of the conductivity depends on the temperature dependence of the mobility of the charge carriers, which in turn depend on the viscosity of the pore water. In this case, the use of an Arrhenius (semiempirical) equation yields

$$
\begin{gathered}
\sigma_{w}(T)=\sigma_{\mathrm{o}} \exp \left(-\frac{E_{a}^{w}}{R T}\right), \\
\sigma_{w}(T)=\sigma_{w}\left(T_{0}\right) \exp \left[-\frac{E_{a}^{w}}{R}\left(\frac{1}{T}-\frac{1}{T_{0}}\right)\right],
\end{gathered}
$$

where $T$ and $T_{0}$ are expressed in degrees $\operatorname{Kelvin}(\mathrm{K})\left(T_{0}=298 \mathrm{~K}\right), \sigma_{\mathrm{o}}$ is a constant that has the dimension of a temperature independent conductivity, $R$ is the universal gas constant $\left(R=8.314 \mathrm{~J} \mathrm{~mol}^{-1} \mathrm{~K}^{-1}\right)$, and $E_{a}^{w}$ (expressed in $\mathrm{J} \mathrm{mol}^{-1}$ ) denotes the activation energy associated with the viscosity of the pore water. For the clean sand alone (experiment 8), the data shown in Figure 10 yield $E_{a}^{w}=14.5 \pm 0.5 \mathrm{~kJ} \mathrm{Mol}^{-1}$. It therefore follows that equations 8 and 9 can be replaced by

$$
\begin{gathered}
\sigma_{\infty}(T)=\sigma_{\infty}\left(T_{0}\right) \exp \left[-\frac{E_{a}^{w}}{R}\left(\frac{1}{T}-\frac{1}{T_{0}}\right)\right], \\
\sigma_{0}(T)=\sigma_{0}\left(T_{0}\right) \exp \left[-\frac{E_{a}^{w}}{R}\left(\frac{1}{T}-\frac{1}{T_{0}}\right)\right],
\end{gathered}
$$

providing some simple and testable expressions for the dependence of the instantaneous and DC conductivities of the mixture with the temperature.

The last parameter to investigate is the Cole-Cole relaxation time (and by extension all of the relaxation times of the system). In Figure $3 \mathrm{c}$ and $3 \mathrm{~d}$, it is clear that there are at least two main relaxation times involved in the system. One $\tau_{e}$ is associated with the formation of the field-induced double layers on each side of the metallic grain ( $e$ stands for external to the metallic grain). 
The second $\tau_{i}$ corresponds to the relaxation time associated with the relaxation of the charge carriers inside the metallic particle ( $i$ stands for inside). In the situation depicted in Figure 3, we have $\tau_{e} \ll \tau_{i}$ (i.e., the polarization is controlled by the relaxation of the charge carriers inside the metallic grains), but this is not necessarily always the case as discussed at the end of this paper (see the "Discussion" section below).

In Revil et al. (2015a; see their discussion), we infer that the characteristic time constant for the polarization process in the electrolyte is given by

$$
\tau_{0}=\frac{a^{2}}{D_{S}}
$$

where $D_{S}$ is the intrinsic diffusion coefficient of the charge carriers in the metallic particles and $a$ is the radius of the metallic particles. The mobility of the charge carriers $\beta_{S}$ can be related to their diffusion coefficients $D_{S}$ through the Einstein relationship $D_{S}=\beta_{S}\left(k_{b} T / e\right)$ with $k_{b}$ being the Boltzmann constant $\left(1.38 \times 10^{-23} \mathrm{~m}^{2} \mathrm{~kg} \mathrm{~s}^{-2} \mathrm{~K}^{-1}\right), T$ is the absolute temperature $(298 \mathrm{~K})$, and $e$ is the elementary charge $\left(1.6 \times 10^{-19} \mathrm{C}\right)$. Using an Arrhenius equation, this yields

$$
D_{S}(T)=D_{0} \exp \left(-\frac{E_{a}}{R T}\right)
$$

where $T$ is expressed in degree Kelvin, $D_{0}$ is proportional to the absolute temperature $T$ and $E_{a}$ denotes the activation energy for the diffusion of the charge carriers that are relaxing in the process of polarization of the metallic particles. Therefore, the temperature dependence of the charge carriers of the grains is given by (see also Appendix A)

$$
D_{S}(T)=D_{S}\left(T_{0}\right) \frac{T}{T_{0}} \exp \left[-\frac{E_{a}}{R}\left(\frac{1}{T}-\frac{1}{T_{0}}\right)\right],
$$

where $T$ and $T_{0}$ are expressed in degree Kelvin $\left(T_{0}=298 \mathrm{~K}\right)$. The time constant obeys therefore the following temperature dependence:

$$
\tau_{0}(T)=\tau_{0}\left(T_{0}\right) \frac{T_{0}}{T} \exp \left[+\frac{E_{a}}{R}\left(\frac{1}{T}-\frac{1}{T_{0}}\right)\right] .
$$

We will see however that equation 14 is unable to explain fully our experimental observations unless a correction factor is applied to this relationship. We will demonstrate later that this correction term, used to explain the dependence of the relaxation time with the salinity, can be justified via finite-element numerical modeling.

We also performed time-domain induced polarization measurements (e.g., Frische and von Buttlar, 1957). We assume now that the primary current $\mathbf{J}_{p}=\sigma_{0} \mathbf{E}$ has been applied from $-T$ to time 0 ( $T$ denotes the period). During the injection of the primary current, each cell of the discretized subsurface will see a secondary current building up. The secondary source current density is determined by (Seigel, 1959)

$$
\mathbf{J}_{S}(t)=-M(t) \mathbf{J}_{p}
$$

Equation 18 means that the dipole moment associated with the polarization of a grain is antiparallel to the applied current density, explaining the sign "-" in this equation. If the period is much greater than all of the relaxation times of the system, the timedependent chargeability (normalized secondary voltage) is given by

$$
M(t)=M\left[1-\sum_{n=0}^{\infty} \frac{(-1)^{n}(1-M)^{n}\left(\frac{t}{\tau_{0}}\right)^{n c}}{\Gamma(1+n c)}\right] .
$$

Equation 19 is written to be consistent with the Cole-Cole complex conductivity model. The function $\Gamma($.$) denotes the Euler gamma$ function defined as
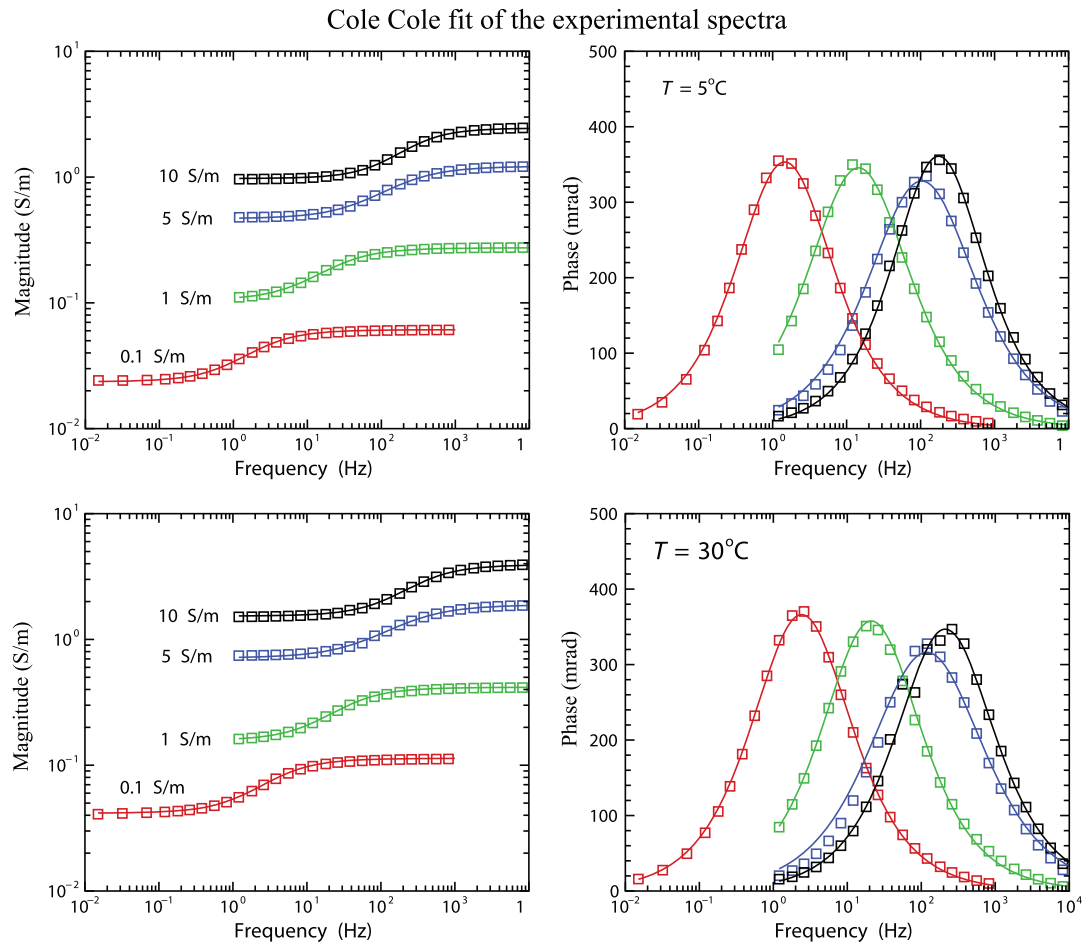

Figure 11. Example of Cole-Cole fit of the complex conductivity spectra (experiments 1 to 4 , big pyrite cube P1). The fit corresponds to the plain lines and is done with the simulated annealing method. 


$$
\Gamma(x)=\int_{0}^{\infty} u^{x-1} e^{-u} d u
$$

where $x>0$. For a period $T$, we can easily use the superposition principle to find the appropriate secondary voltage response function (see Tombs, 1981). Note that equation 19 is built on the timedomain solution for the Pelton complex resistivity model (Pelton et al., 1978) in which we replaced the Pelton time constant $\tau_{P}$ by the time constant for the complex conductivity model, i.e., $\tau_{0}$, using $\tau_{0}=\tau_{P}(1-M)^{1 / c}$ (see Florsch et al., 2012; Tarasov and Titov, 2013, their equation 24).

\section{Fit of the data}

The frequency-domain and time-domain measurements were inverted to determine the four Cole-Cole parameters (i.e., $\sigma_{\infty}$, $\sigma_{0}, \tau$, and $c$ ) using a stochastic approach. For the frequency-domain measurements, we use the magnitude and phase of the complex conductivity measurements at different frequencies were used in the objective function to define a data misfit term using the L2-norm (there is no regularization term involved). The same procedure was followed for the time-domain measurements using the secondary voltage decay a given period. For the frequency-domain measurements, equal weights were assigned for the magnitude and phase measurements in the inversion of the model parameters at different equilibrium temperatures. During SIP measurements, 13 data points were taken in each decade. With the frequency range from 0.01 to $1000 \mathrm{~Hz}$, there are definitely more data than the four unknown parameters. As shown, for instance, by Ghorbani et al. (2007), the Cole-Cole parameter inversion using frequency-domain data is generally presenting a well-posed problem. For time-domain data, several input voltage or current periods need to be used to reduce the nonunicity of the inversion.

The inversion was done with the simulated annealing method using the "simulannealbnd" function in MATLAB used as a global optimization scheme. We used constraints on the four Cole-Cole
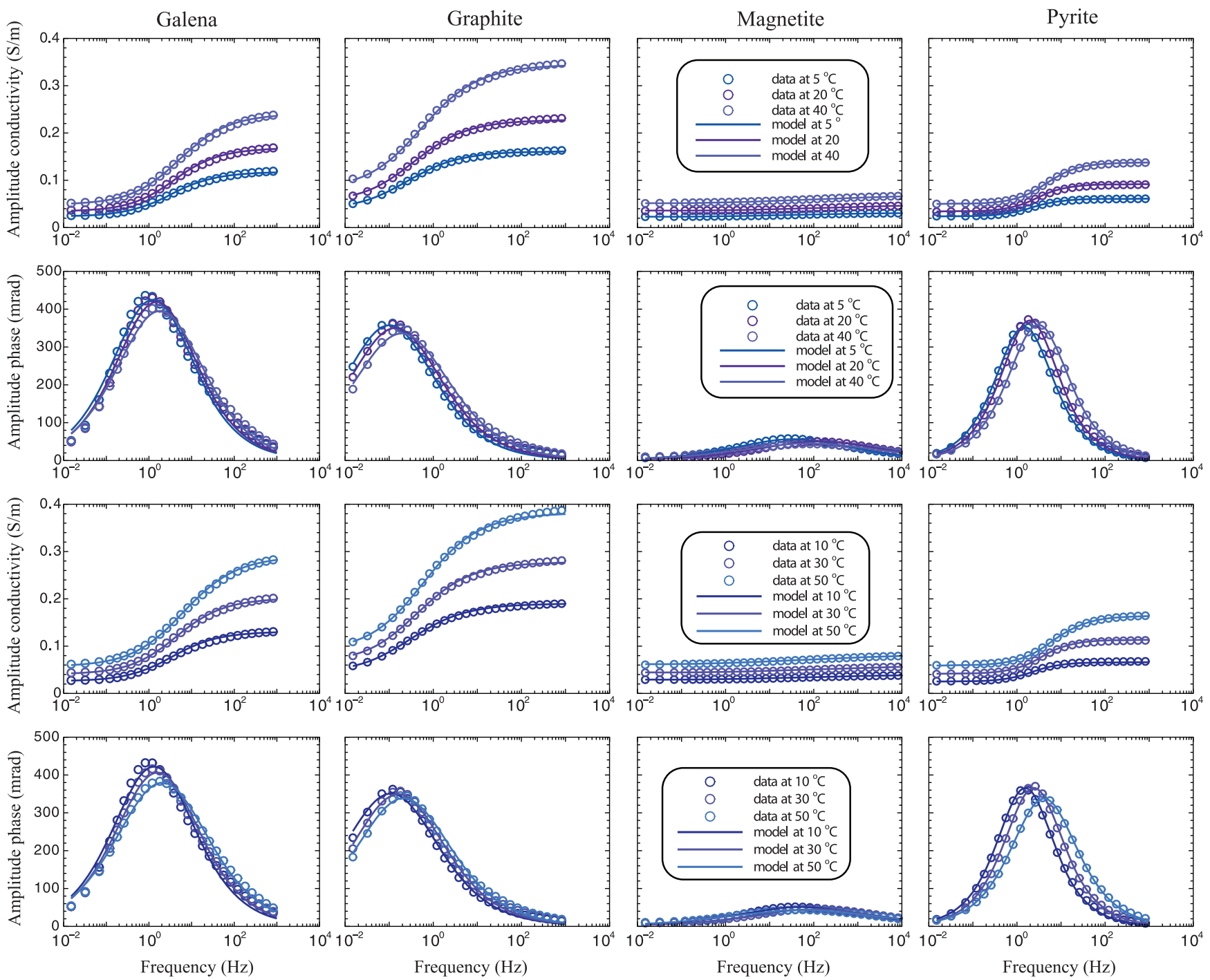

Figure 12. Example of Cole-Cole fit of the complex conductivity spectra (experiments 1-P1, 6-M1, 7-Gr2, and 9-G1). The fit corresponds to the plain lines and is done with the simulated annealing method as explained in the main text. 
parameters based on the raw data themselves. Indeed, from the raw data (see, for instance, Figure 5), we can easily determine a range of values for $\sigma_{\infty}$ (from the high-frequency asymptotic limit of the inphase conductivity), $\sigma_{0}$ (from the low-frequency asymptotic limit of the in-phase conductivity), and $\tau$ (from the phase peak frequency). For the Cole-Cole exponent, we limit the search to the range [0.5-1]. We have checked that these choices speed up the search of the global minimum of the cost function by the simulated annealing method, but they do not change the optimum values. In the simulannealbnd function, the default convergence condition is the objective function smaller than $10^{-6}$ and the step between two iterations larger than $10^{-10}$. Stochastic inversion of the Cole-Cole parameters is known to be a robust approach using frequency-domain data (Chen et al., 2008). Examples of the fit of the spectra with the Cole-Cole complex conductivity model are shown in Figures 11 and 12 . We see that the complex conductivity model is clearly able to represent the data (one exception is the chalcopyrite, which seems to have a phase peak at too-low frequencies). The Cole-Cole parameters resulting from this fitting procedure are shown in Figures 13, 14, 15, 16, 17, 18, and 19.

\section{RESULTS}

\section{Effect of temperature}

Some characteristic dependences of the Cole-Cole exponent with the temperature are shown in Figures 13 and 14. As expected from the discussion given in the previous section, the Cole-Cole exponent $c$ is temperature independent. Similarly, the characteristic dependence of the chargeability with the temperature is shown in Figures 13 and 15. As expected again from the discussion given in the previous section, the chargeability $M$ is temperature independent. As shown in Figure 4, the chargeability depends on the amount of metallic particles in the material plus the background chargeability, both being temperature independent.

In Figures 13, 16, and 17, we show that the temperature dependence of the DC conductivity $\sigma_{0}$ and instantaneous conductivity $\sigma_{\infty}$
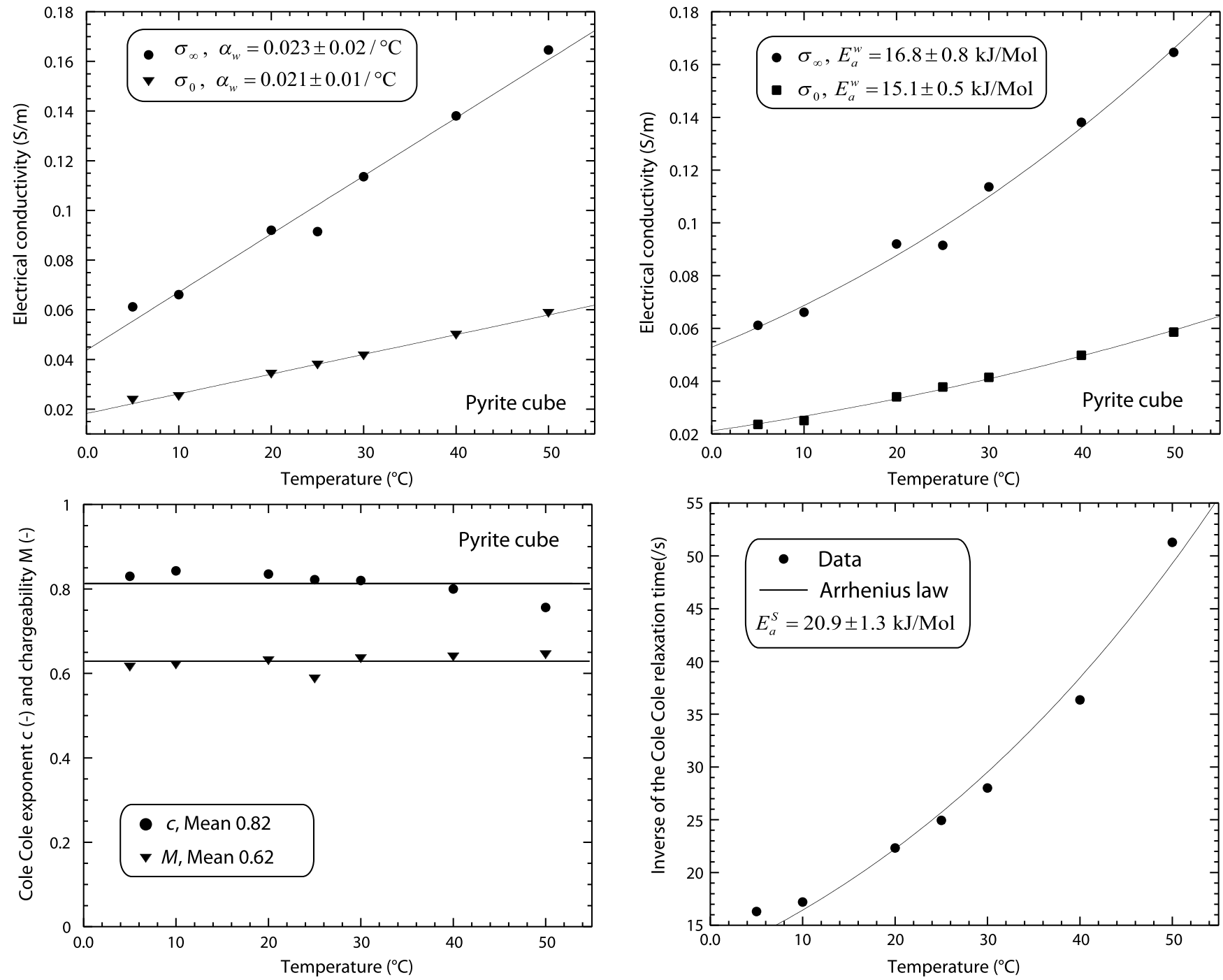

Figure 13. Temperature dependence of the instantaneous conductivity $\sigma_{\infty}$, steady-state conductivity $\sigma_{0}$ (see Figure 3), Cole-Cole exponent $c$, chargeability $M$, and Cole-Cole relaxation time $\tau_{0}$ for the big pyrite cube P1 determined from the inversion of the data in experiment 1 . Note that the Cole-Cole exponent and the chargeability do not depend on the temperature in agreement with the theory. 
are identical. The data can be fitted by an Arrhenius law with an activation energy comprised between 13 and $15 \mathrm{~kJ} \mathrm{Mol}^{-1}$. From Table 3, the mean values and standard deviations for the DC conductivity $\sigma_{0}$ and instantaneous conductivity $\sigma_{\infty}$ are $14.0 \pm 1.1$ and $14.0 \pm 1.4 \mathrm{~kJ} \mathrm{Mol}^{-1}$, respectively. This is consistent with the activation energy of the pore water of clean sand $14.5 \pm 0.5 \mathrm{~kJ} \mathrm{Mol}^{-1}$ (see Table 3). For water, the activation energy reported for the viscosity is typically at approximately $15.6-15.8 \mathrm{~kJ} \mathrm{Mol}^{-1}$ (e.g., Viktor and Hazra, 2002; Toumi et al., 2009). The activation energy does not depend on the salinity. Therefore, the temperature dependence of the DC conductivity $\sigma_{0}$ and instantaneous conductivity $\sigma_{\infty}$ agrees with the temperature dependence of the pore-water viscosity as expected.
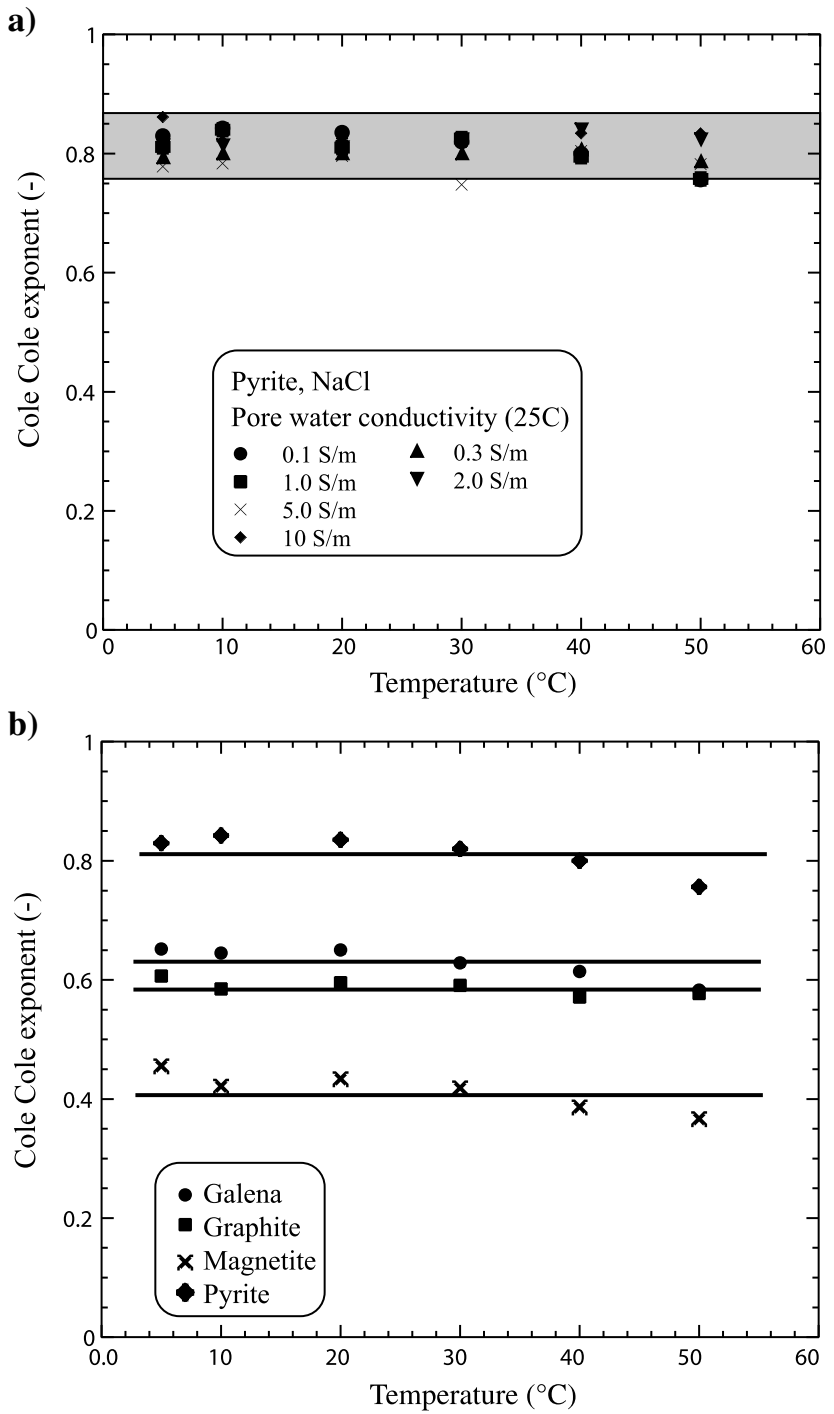

Figure 14. Evolution of the Cole-Cole exponent $c$ for the complex conductivity model for some core samples. The Cole-Cole exponent appears essentially temperature independent. (a) Experiments with the big pyrite cube P1 at six different salinities (experiments 1 to 4 and 14 and 15). (b) Experiment with different types of mineralizations. Experiment 1 (pyrite), experiment 6 (magnetite), experiment 7 (graphite), and experiment 9 (galena).
The last point to investigate is the temperature dependence of the relaxation time (see Figures 18 and 19). It can be roughly fitted with an Arrhenius law. From Table 3, the mean values and standard deviations for the Cole-Cole relaxation time are 7.9 $\pm 4.2 \mathrm{~kJ} \mathrm{Mol}^{-1}$ (the mean value and standard deviation for pyrite alone are $5.7 \pm 2.0 \mathrm{~kJ} \mathrm{Mol}^{-1}$; see Figure 19a). These values are therefore clearly smaller than the activation energy of the DC and instantaneous conductivities, and it shows that the polarization process is not controlled by the formation of the field-induced DL around the metallic particles but possibly by the polarization of the metallic particles themselves (see Figure 3). The dependence of the ColeCole relaxation time of graphite at two salinities is shown in
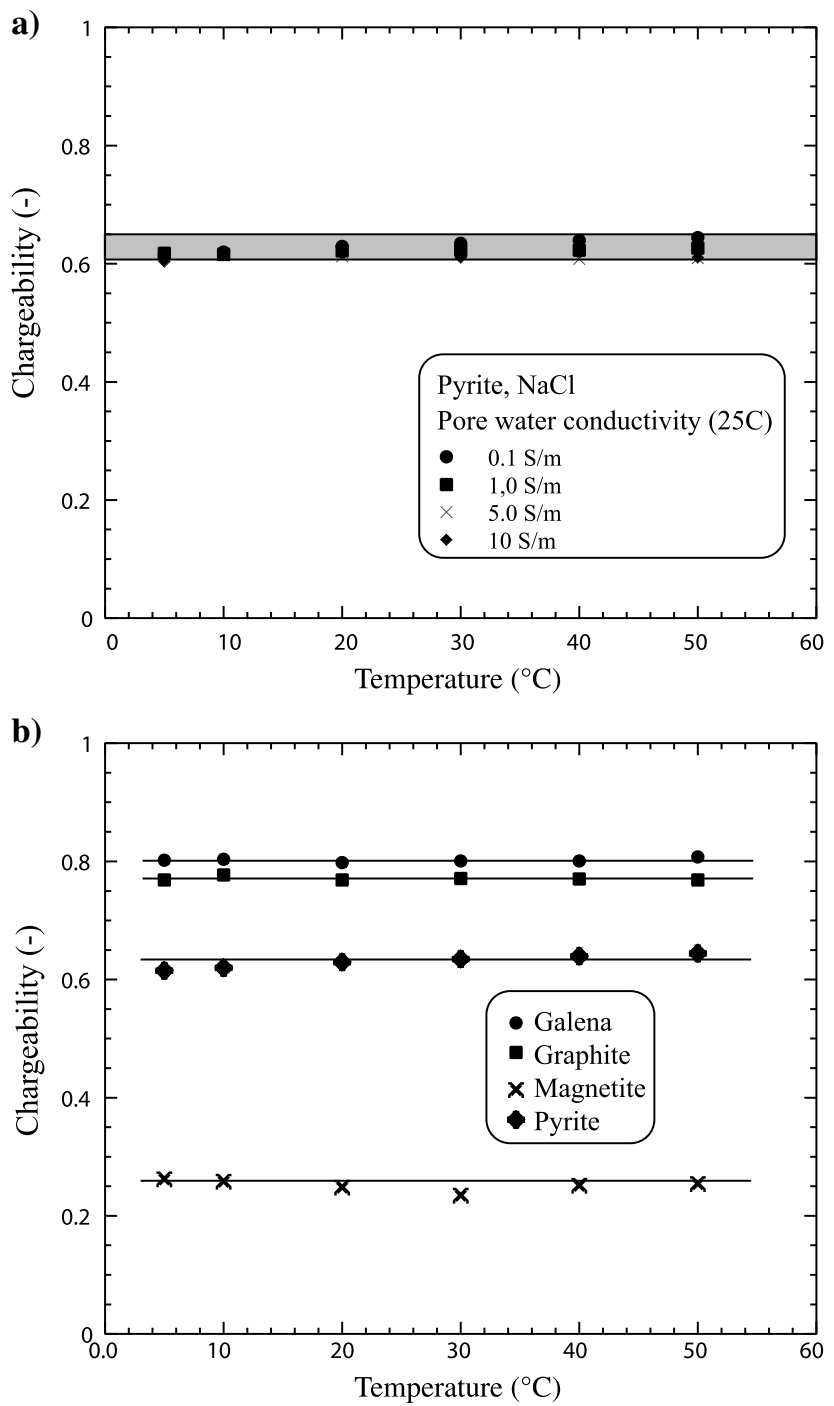

Figure 15. Evolution of the chargeability $M$ for the complex conductivity model for some core samples. The chargeability appears essentially temperature independent. (a) Experiments with pyrite cube P1 at four different salinities (experiments 1 to 4). (b) Experiments with different mineralizations. Experiment 1 (pyrite), experiment 6 (magnetite), experiment 7 (graphite), and experiment 9 (galena). 
Figure $19 \mathrm{~b}$. The trends are characterized by a higher activation energy $11 \pm 2 \mathrm{~kJ} \mathrm{Mol}^{-1}$.

\section{Effect of salinity on the Cole-Cole parameters}

Figure 20a shows that the chargeability and the Cole-Cole exponent are independent of the salinity in agreement with the theory and with the experimental data recently presented by Abdulsamad et al. (2017). Figure 20b shows that the DC and instantaneous conductivities of the mixture depend linearly on the conductivity of the pore water. This is expected from the theory. Indeed, considering equations 3 and 4 and a background conductivity given by the conductivity of the pore water divided by the formation factor of the sand, we have

$$
\sigma_{\infty} \approx \frac{\sigma_{w}}{F_{s d}}\left(1+3 \varphi_{m}+\cdots\right)
$$
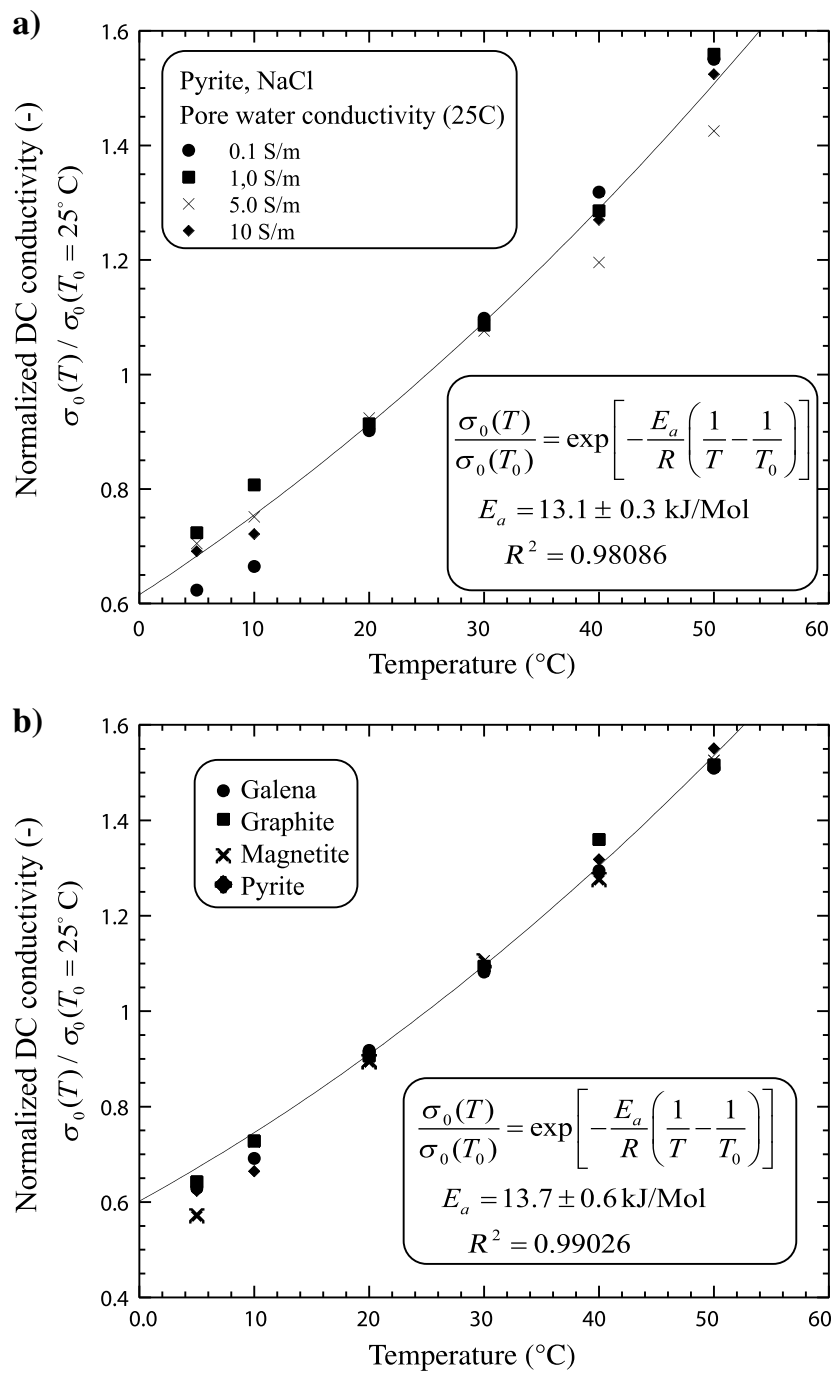

Figure 16. Evolution of the normalized DC conductivity with temperature for some of the core samples. The dependence on temperature of the DC conductivity can be fitted with an Arrhenius law. (a) Experiments with big pyrite cube P1 at four different salinities (experiments 1 to 4). (b) Experiments with different mineralizations. Experiment 1 (pyrite), experiment 6 (magnetite), experiment 7 (graphite), and experiment 9 (galena).

$$
\sigma_{0} \approx \frac{\sigma_{w}}{F_{s d}}\left(1-\frac{3}{2} \varphi_{m}+\cdots\right),
$$

where $F_{s d}$, the intrinsic formation factor of the sand, is reported in Table $1\left(F_{s d}=3.7\right)$. We could define the low-frequency and high-frequency formation factors as

$$
\begin{gathered}
\sigma_{\infty} \approx \frac{\sigma_{w}}{F_{\infty}}, \\
\sigma_{0} \approx \frac{\sigma_{w}}{F_{0}},
\end{gathered}
$$
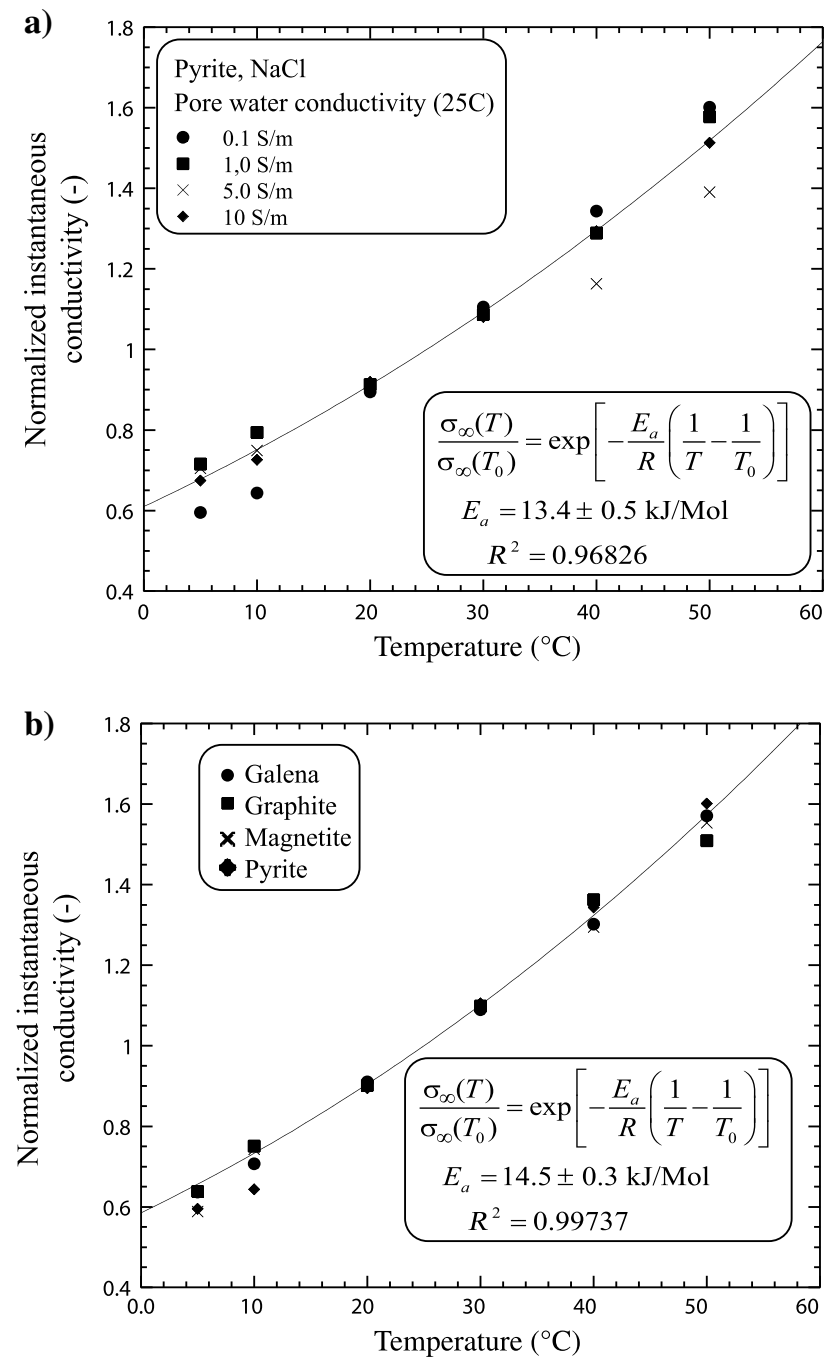

Figure 17. Evolution of the normalized instantaneous conductivity of the complex conductivity model for some core samples. The dependence on temperature of the instantaneous conductivity can be fitted with an Arrhenius law. (a) Experiments with pyrite cube P1 at four different salinities (experiments 1 to 4). (b) Experiments with different mineralizations. Experiment 1 (pyrite), experiment 6 (magnetite), experiment 7 (graphite), and experiment 9 (galena). 
where $F_{\infty}=F_{s d} /\left(1+3 \varphi_{m}+\cdots\right)$ and $F_{0}=F_{s d} /\left(1-1.5 \varphi_{m}+\cdots\right)$. For the experiments reported in Figure 20b, we have $\varphi_{m}=0.26$, which yields the following experimental values: $F_{\infty}=3.03$ and $F_{0}=7.09$. We can use the measured values of $F_{\infty}$ and $F_{0}$ to determine the values of $\varphi_{m}$ and $F_{s d}$. Using

$$
\begin{gathered}
\varphi_{m}=\frac{F_{0}-F_{\infty}}{3\left(F_{\infty}+F_{0} / 2\right)}, \\
F_{S d}=F_{\infty}\left(1+3 \varphi_{m}\right) .
\end{gathered}
$$

This yields $\varphi_{m}=0.21$ and $F_{s d}=4.9$. These values are close to the measured values $\varphi_{m}=0.26$ and $F_{s d}=3.7$ (see Table 1).
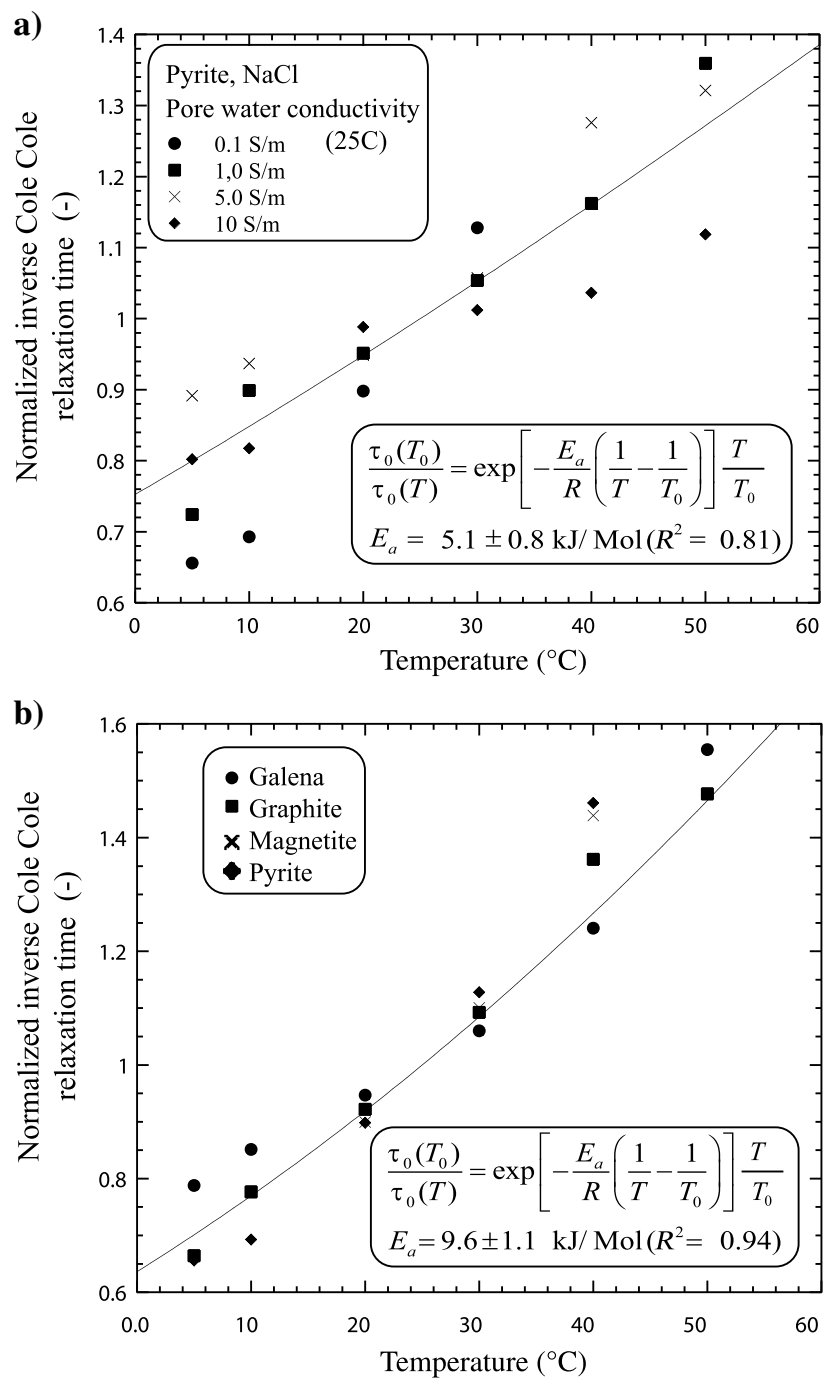

Figure 18. Evolution of the inverse of the Cole-Cole relaxation time of the complex conductivity model for some core samples. The dependence on temperature of the Cole-Cole relaxation time can be fitted with an Arrhenius law. (a) Experiments with pyrite cube P1 at four different salinities (experiments 1 to 4 ). The dispersion in the results implies that in reality the activation energy depends on the conductivity of the pore water. (b) Experiments with different mineralizations. Experiment 1 (pyrite), experiment 6 (magnetite), experiment 7 (graphite), and experiment 9 (galena).
Regarding the effect of salinity, Figure 21 shows that the ColeCole relaxation time is inversely proportional to the conductivity of the pore water. Hupfer et al. (2016) formulate the inverse proportionality between the time constant and the conductivity of the pore water in a similar way. Such a dependence is however not predicted by the model described above. A similar work was done for the graphite sample Gr2, and the relaxation time shows also an inverse dependence to the conductivity of the pore water at a constant temperature.

\section{Time-domain induced polarization}

Figure 22 shows the normalized secondary voltage versus time at six different temperatures for the big pyrite cube P1 in sand. The data were fitted by a Cole-Cole model (plain lines in Figure 22). For this experiment, the chargeability and the Cole-Cole exponent do not change with the temperature. The uncertainty regarding the determination of the relaxation time $\tau_{0}$ was too big to determine precisely the temperature dependence of this Cole-Cole parameter with temperature.

\section{NUMERICAL SIMULATIONS}

Based on the solution of the Poisson-Nernst-Planck equations, Abdulsamad et al. (2017) recently present a numerical simulation of the polarization phenomena in a background medium containing semiconductor materials. The fundamental equations are summarized in Appendix B. In this simulation, the unique source of polarization is the electromigration of the charge carriers and their accumulation at both sides of the metallic-electrolyte interface. No oxidation-reduction reactions at the interface between the metallic particles and the background material are considered. The numerical simulations are based on the finite-element method (for details, see Abdulsamad et al., 2017). Our goal is to see if these simulations can be extended to see the effect of salinity and temperature on the spectra. To simplify the problem, we consider a pyrite grain of small size $(20 \mu \mathrm{m}$ in grain radius) immersed in a background electrolyte with $\mathrm{NaCl}$ (Figure 23). The small size of the metallic grain is imposed by numerical constraint in our study because of meshing restrictions.

The observed dependence of the relaxation time on the salinity could be explained by considering the semiconductor grain as an electrical dipole formed during the current injection. This electrical dipole impacts the electric potential and consequently the charge distribution in its vicinity. Figure 24 shows the variation of the charge concentration away from the metallic particle/electrolyte interface at the end of the current injection time. We notice that, as long as the electrolyte concentration is low, the influence of the electric dipole is further from the grain/electrolyte interface, which provides a higher relaxation time (a larger distance for the ions to go back to the equilibrium state). These results are qualitatively consistent with the experimental observation of the relaxation time dependence with salinity at different temperatures.

On the other hand, to test the dependence of the numerical response with temperature, we fixed all of the parameters (see Table 4) and we changed the temperature in the same range tested with our laboratory measurements. Changing the temperature affects the diffusion coefficients in the semiconductor and in the electrolyte. The diffusion coefficient changes with temperature according to the Arrhenius law (equation 16) with an activation energy of $15 \mathrm{~kJ} \mathrm{~mol}^{-1}$ for the ions. Figure 25 shows the dependence of the secondary 
voltage versus time at different temperatures. The relaxation time calculated from the numerical simulation depends on temperature as shown in Figure 26. We notice that the relaxation time decreases slightly (in the same order of magnitude) with temperature. The small values of the relaxation time calculated from this simulation come from the small grain radius $(20 \mu \mathrm{m})$. For these small grain sizes, the polarization is controlled by the formation of DLs generated by the application of the primary electrical field. That said, such type of polarization does not dominate the observed response for larger metallic particles as discussed in more detail in the next section. With the exception of the relaxation time, the results of the numerical simulation are qualitatively in agreement with our experimental results for the other Cole-Cole parameters.

\section{DISCUSSION}

In this discussion, we want to come back to the two relaxation times of the problem sketched in Figure $3, \tau_{e}$ and $\tau_{i}$, i.e., corresponding to the relaxation of the charge carriers in the electrolyte (ions for the electrical field-induced electrical DLs) and the relaxation time associated with the electrodiffusion of the charge carriers in the metallic particles (electrons and p-holes), respectively. We are going to compare these two relaxation times as a function of the properties of the problem. The relaxation time associated with the electrical-field-induced electrical DLs is given by (see Chu and Bazant, 2006)

$$
\tau_{e}=\frac{a x_{d}}{2 D_{( \pm)}},
$$

where $D_{( \pm)}$denotes the diffusion coefficient of the ions in the electrolyte around the metallic particles and $x_{d}$ denotes the Debye length scale defined by

$$
x_{d}=\sqrt{\frac{k_{b} T \varepsilon_{w}}{2 e^{2} C_{( \pm)}^{0}}} .
$$

In equation $28, e$ denotes the elementary charge $\left(1.6 \times 10^{-19} \mathrm{C}\right), k_{b}$ is the Boltzmann constant $\left(1.38 \times 10^{-23} \mathrm{~m}^{2} \mathrm{~kg} \mathrm{~s}^{-2} \mathrm{~K}^{-1}\right), \varepsilon_{w}$ denotes the dielectric constant of the pore water (see Table 4), $C_{(+)}^{0}$ is the concentration of the electrolyte (in ions per $\mathrm{m}^{3}$ ), and $T$ is the absolute temperature (in K). For the concentration, we can use a concentration in $\mathrm{MolL}^{-1}$ by replacing $C_{( \pm)}^{0}$ by $10^{3} N C_{( \pm)}^{0}$, where $N$ denotes the Avogadro number $\left(6.022 \times 10^{23} \mathrm{Mol}^{-1}\right)$. The Debye length provides an estimate of the thickness of the DL formed around the metallic particle (typically in the range of 10-100 nm).

The relaxation time associated with the relaxation of the charge carriers inside the metallic particle is given by (Revil et al., 2015a)

$$
\tau_{i}=\frac{a^{2}}{D_{m}} .
$$

The relaxation time associated with the internal polarization of the charge carriers of the metallic particle is much larger than the relaxation time of the ions in the field-induced electrical DL (i.e., $\left.\tau_{e} \ll \tau_{i}\right)$ if the following condition is met:

$$
a \gg \frac{D_{m}}{2 D_{( \pm)}} x_{d} .
$$

Table 3. Properties of the spectra for the recorded experiments. The parameter $c$ denotes the Cole-Cole exponent, $M$ is the mean chargeability (averaged over the temperature range), the activation energy $E_{a}^{w}$ is determined from the temperature dependence of the instantaneous and DC conductivities, and the activation energy $E_{a}$ is determined from the activation energy of the relaxation time. Experiment 11 (C2-Chalcopyrite) could not be fitted with a Cole-Cole model, and the results are not reported here. For experiment 8 (pure sand), the activation energy for the conductivity is $14.5 \pm 0.5 \mathrm{~kJ} \mathrm{Mol}^{-1}$. For the time-domain measurements, the DC conductivity is determined first and the instantaneous conductivity is determined from the DC conductivity and the chargeability.

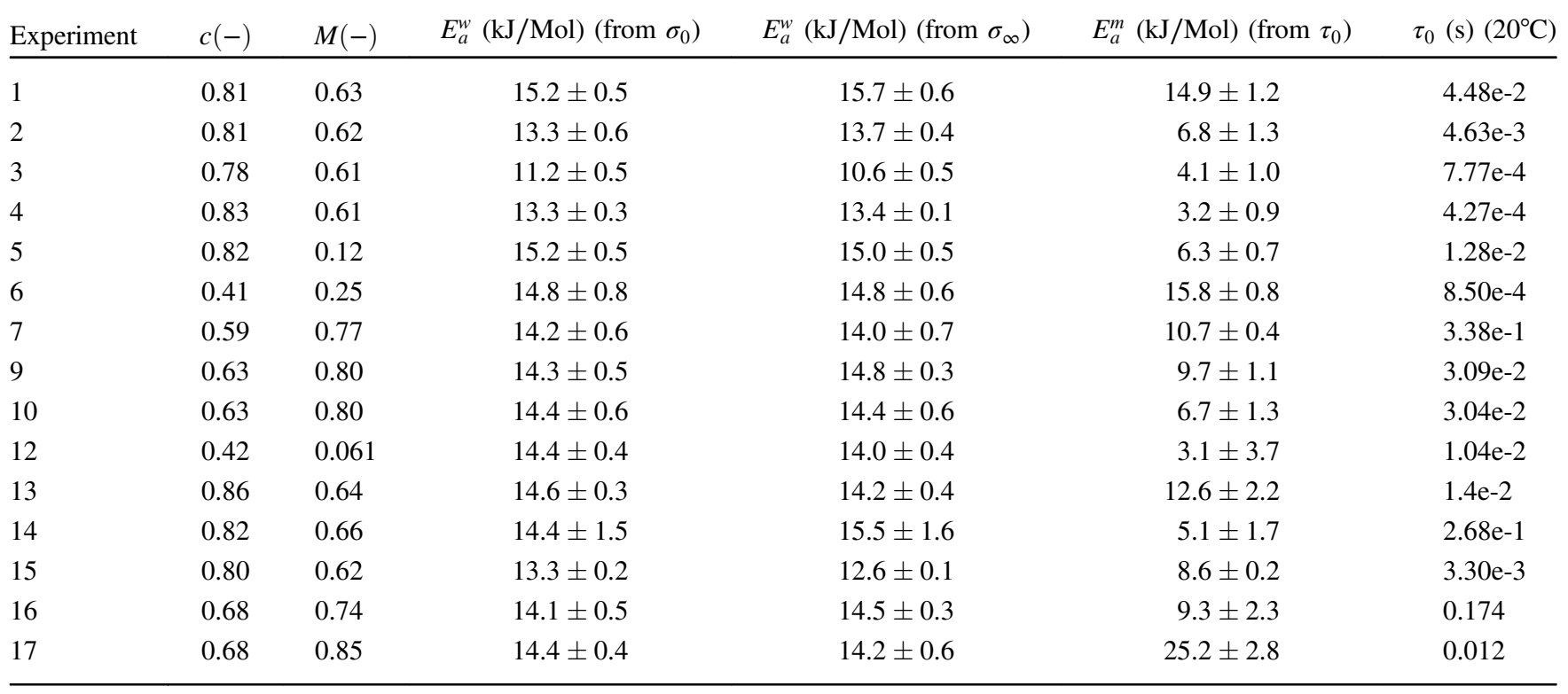


Taking $D_{m}=10^{-5} \mathrm{~m}^{2} \mathrm{~s}^{-1}, D_{( \pm)}=10^{-9} \mathrm{~m}^{2} \mathrm{~s}^{-1}$ (see Table 4) and $x_{d}=100 \mathrm{~nm}$ (at low salinities), we have $a \ll 500 \mathrm{~mm}$. For the minerals investigated in the laboratory in the present study, this is always the case. In other words, the polarization of the mixtures is always controlled by the polarization of the charge carriers inside the metallic grains. That said, for the numerical experiment described in the previous section, we choose (because of computational constraints) a small size for the metallic particle $(20 \mu \mathrm{m})$. In these conditions, the polarization was controlled by the polarization of the charge carriers outside the metallic particles, explaining that the temperature dependence of the relaxation time was controlled by the temperature dependence of the diffusion coefficient of the ion charge carriers and characterized by an activation energy of approximately $14 \mathrm{~kJ} \mathrm{Mol}^{-1}$. This partly solves the discrepancy observed above
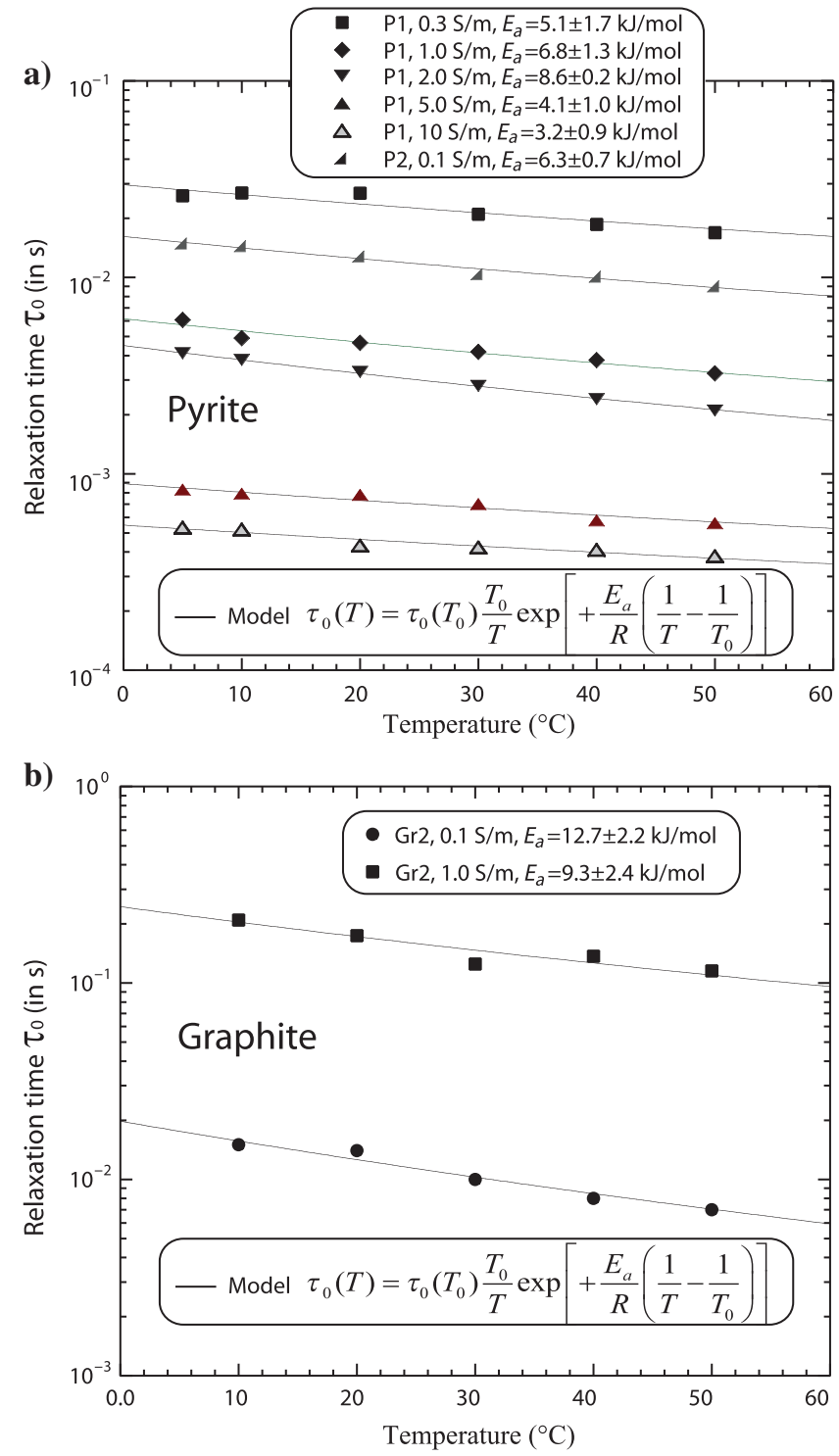

Figure 19. Dependence of the Cole-Cole relaxation time with temperature. (a) Experiments 2 to 5, 14, 15, pyrite cubes. Note that it is more difficult to precisely determine the value of the activation energy when its value is small. The mean value and standard deviation are $5.7 \pm 2.0 \mathrm{~kJ} \mathrm{Mol}^{-1}$. (b) Experiments 7 and 16, graphite Gr2. between the numerical experiments and the experimental data. The fact that at a small particle radius of metallic particle, the relaxation time is directly proportional to the particle radius (rather than the square of the particle radius) is consistent with the finite-element simulations of Abdulsamad et al. (2017, their Figure 13).

Finally, according to equation 30 , for large grains $(>500 \mu \mathrm{m}$ at low salinity), we can neglect the formation of field-induced DLs. We have therefore done a last set of numerical simulations neglecting this effect and keeping only the polarization due to the electrodiffusion of the charge carriers inside the metallic grain. For a grain with a radius of $1.5 \mathrm{~cm}$, the relaxation time is on the order of a second. It decreases with the increase of the conductivity of the
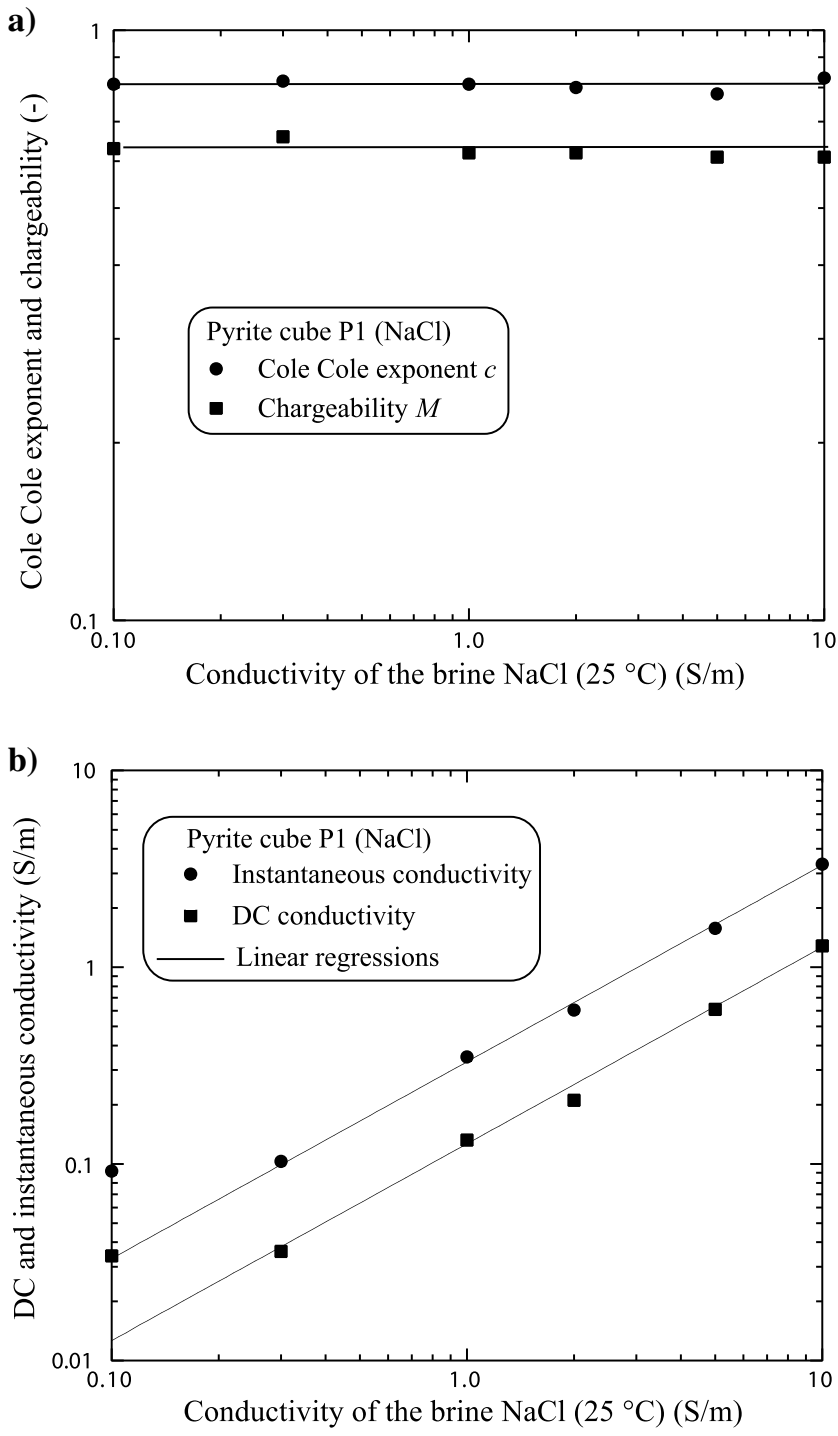

Figure 20. Cole-Cole parameters as a function of the conductivity of the brine at $25^{\circ} \mathrm{C}$. (a) Cole-Cole exponent and chargeability versus salinity. We use an average of all the values determined in the temperature range $5^{\circ} \mathrm{C}-50^{\circ} \mathrm{C}$. Experiments 1 to 4 and 14 and 15 . (b) Dependence on the $\mathrm{DC}$ and instantaneous conductivities at $20^{\circ} \mathrm{C}$ as a function of the conductivity of the brine. The slopes of these linear trends yield the following values for the apparent formation factors: $F_{\infty}=3.03$ and $F_{0}=7.09$. 
background electrolyte and increases with the increase of the metallic particle conductivity itself (see Figure 27). The conductivity of the metallic grains is controlled, at a given temperature, by the concentration of the charge carriers. This dependence can be written as

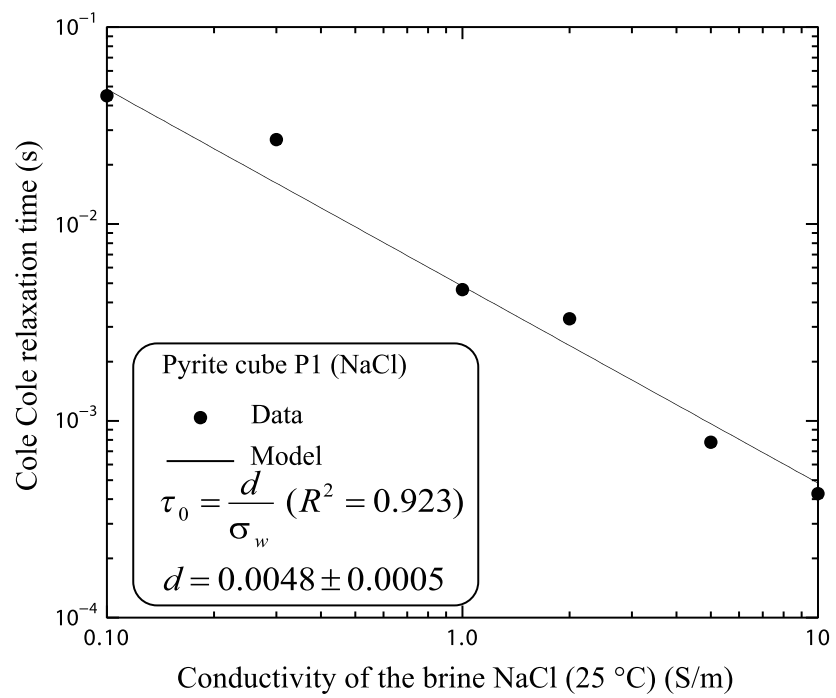

Figure 21. Cole-Cole relaxation time (in s) as a function of the conductivity of the brine at $25^{\circ} \mathrm{C}$. Experiments 1 to 4 and 14 and 15 . It appears that at a given temperature $\left(20^{\circ} \mathrm{C}\right)$, the relaxation time is inversely proportional to the conductivity of the brine expressed in $\mathrm{S} \mathrm{m}^{-1}$. For graphite (sample $\mathrm{Gr} 2$ ), the relaxation time follows a similar inverse relationship with $d=0.07 \mathrm{smS}^{-1}$. In this plot, $d$ denotes a constant of proportionality between the relaxation time and the inverse of the conductivity of the pore water (at a constant temperature).

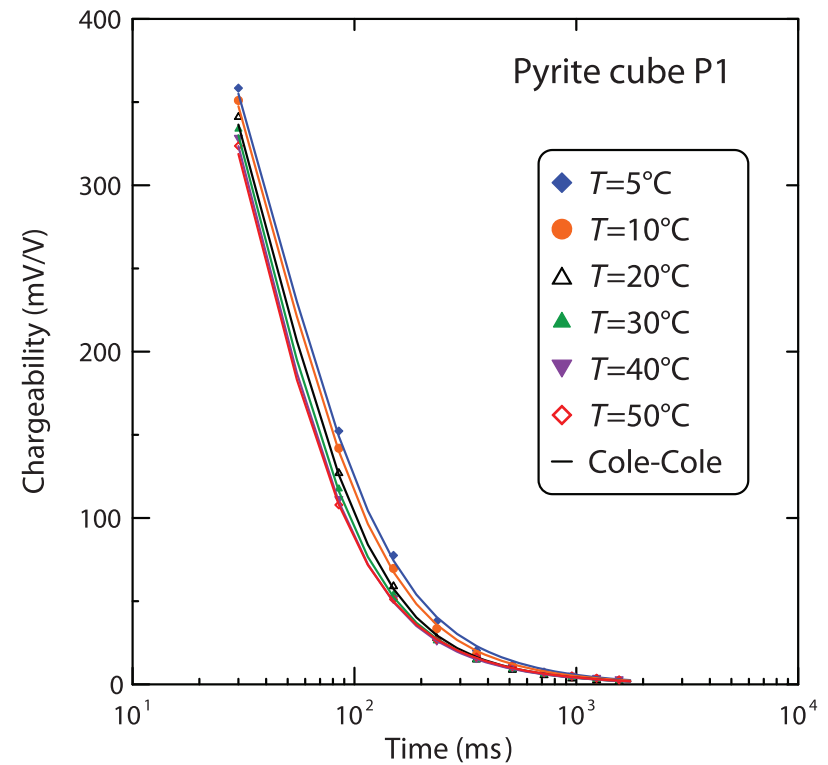

Figure 22. Temperature dependence of the time-domain induced polarization data. Dependence of the secondary voltage relaxation curves with the temperature for the big pyrite cube P1 (experiment 13 ). The primary current is turned on for $2 \mathrm{~s}\left(T_{\mathrm{on}}\right)$. This curve can be fitted with a Cole-Cole model (solid lines) with $M=0.64 \pm 0.04$ and $c=0.85 \pm 0.02$.

$$
\tau_{i}=\frac{a^{2}}{D_{m}}\left(1+\frac{\sigma_{S}^{\infty}}{\sigma_{w}}\right),
$$

where $\sigma_{S}^{\infty}$ denotes the instantaneous conductivity of the metallic grain. Equation 31 should replace equation 14 in our previous model. When the ratio $\sigma_{S}^{\infty} / \sigma_{w}$ is much larger than one, we have

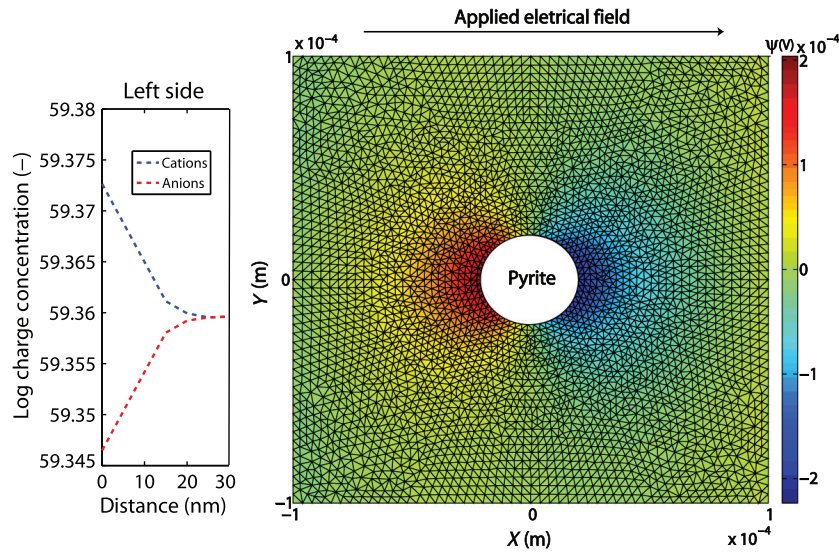

Figure 23. Numerical modeling. (a) Two-dimensional finiteelement mesh used in the numerical simulations and computation of the secondary potential distribution $\psi(r)$ showing the dipolar moment created by the metallic particle (grain radius $20 \mu \mathrm{m}$ ) immersed in a background electrolyte of salinity $0.1 \mathrm{~mol} \mathrm{~L}^{-1}$. (b) Distribution of the electrical potential during a cycle in the application of the electrical field and for different temperatures. (c) Distribution of cations and anions in the vicinity of the left side of the metallic particles (the distance is taken from the surface of the metallic particle). We observe therefore an excess of cations and a deficiency of anions on this side of the grain and the opposite (not explicitly shown here) on the other side in agreement with Figure 3. This corresponds to the formation of field-induced DLs on each side of the metallic grain.

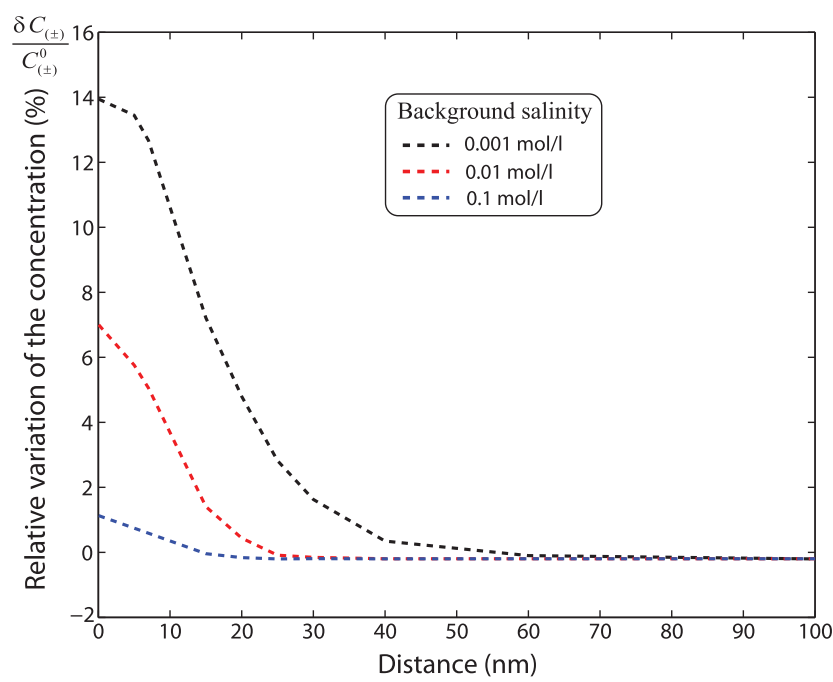

Figure 24. Variation of the concentration of the ionic species in the vicinity of the mineral water interface $(d=0$ on the mineral surface) at the end of the primary current injection. We observe that at high salinities, the relative change in the ionic concentrations close to the surface of the metallic particle is smaller than at low salinities. 


$$
\tau_{i} \approx \frac{a^{2} \sigma_{S}^{\infty}}{D_{m} \sigma_{w}}
$$

Because $\sigma_{S}^{\infty}$ is expected to be proportional to the diffusion coefficient of the charge carriers, the relaxation time is expected to be independent of the diffusion time of the charge carriers. Because the mobility of the charge carriers and their diffusion coefficient are related to each other through the Einstein relationship $D_{s}=\left(\beta_{s} k_{b} T / e\right)$ with $k_{b}$ the Boltzmann constant $\left(1.38 \times 10^{-23} \mathrm{~m}^{2} \mathrm{~kg} \mathrm{~s}^{-2} \mathrm{~K}^{-1}\right), \quad T$ is the absolute temperature

Table 4. Parameters used for finite-element simulation. The quantity $C_{( \pm)}$denotes the ionic concentration at equilibrium state, $C_{( \pm)}^{m}$ denotes the charge concentration in the semiconductor grain at equilibrium state, $D_{( \pm)}$denotes the diffusion coefficients of the ionic species in the surrounding of the metallic particle, $D_{(+)}^{m}$ denotes the diffusion coefficients of the charge carriers in the metallic particle, and $\varepsilon_{0}, \varepsilon_{S}$, and $\varepsilon_{w}$ denote the permittivity of free space, the permittivity of pyrite, and the permittivity of electrolyte, respectively.

Parameter

\begin{tabular}{lc}
\hline$D_{(+)}=D_{(-)}$ & $1.2 \times 10^{-9} \mathrm{~m}^{2} \mathrm{~s}^{-1}$ \\
$D_{(+)}^{m}=D_{(-)}^{m}$ & $2.9 \times 10^{-5} \mathrm{~m}^{2} \mathrm{~s}^{-1}$ \\
$C_{(+)}^{m}=C_{(-)}^{m}$ & $5 \times 10^{23} \mathrm{~m}^{-3}$ \\
$C_{(+)}=C_{(-)}$ & $0.1 \mathrm{~mol} \mathrm{~L}^{-1}$ \\
$T_{0}$ & $298 \mathrm{~K}$ \\
$\varepsilon_{0}$ & $8.81 \times 10^{-12} \mathrm{~F} / \mathrm{m}$ \\
$\varepsilon_{S}$ & $10.9 \varepsilon_{0}$ \\
$\varepsilon_{w}$ & $80 \varepsilon_{0}$ \\
\hline
\end{tabular}

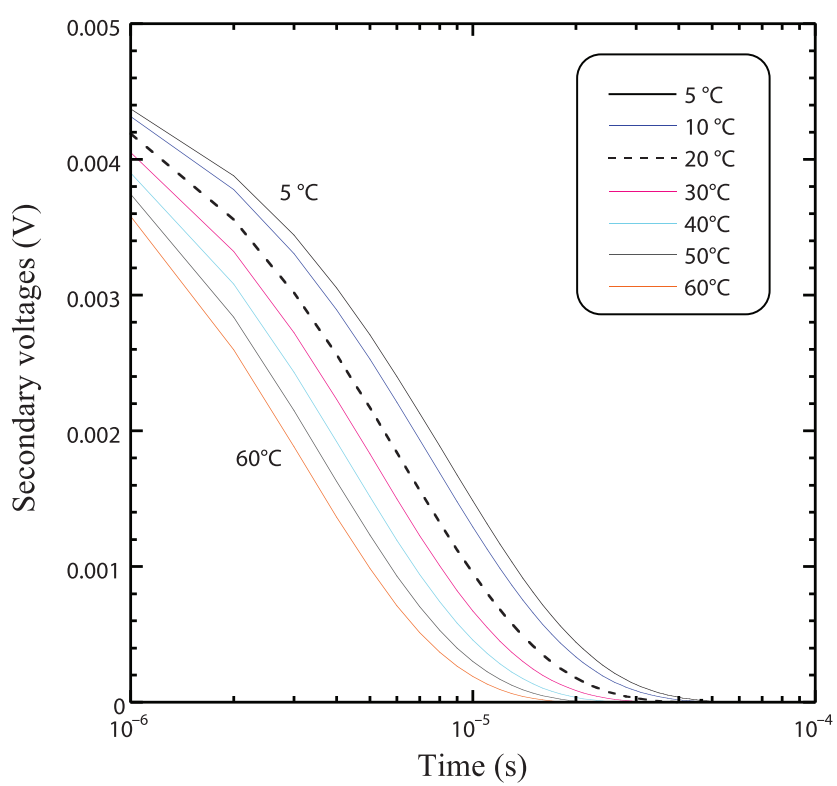

Figure 25. Dependence of the secondary voltage relaxation curves with the temperature. The primary current is turned on for $0.05 \mathrm{~ms}$ $\left(T_{\text {on }}\right)$. This curve can be fitted with a Debye model and a chargeability that is independent of the temperature.
$(298 \mathrm{~K})$, and $e$ is the elementary charge $\left(1.6 \times 10^{-19} \mathrm{C}\right)$, we obtain

$$
\tau_{i} \approx \frac{a^{2} e^{2} C_{m}}{k_{b} T \sigma_{w}},
$$

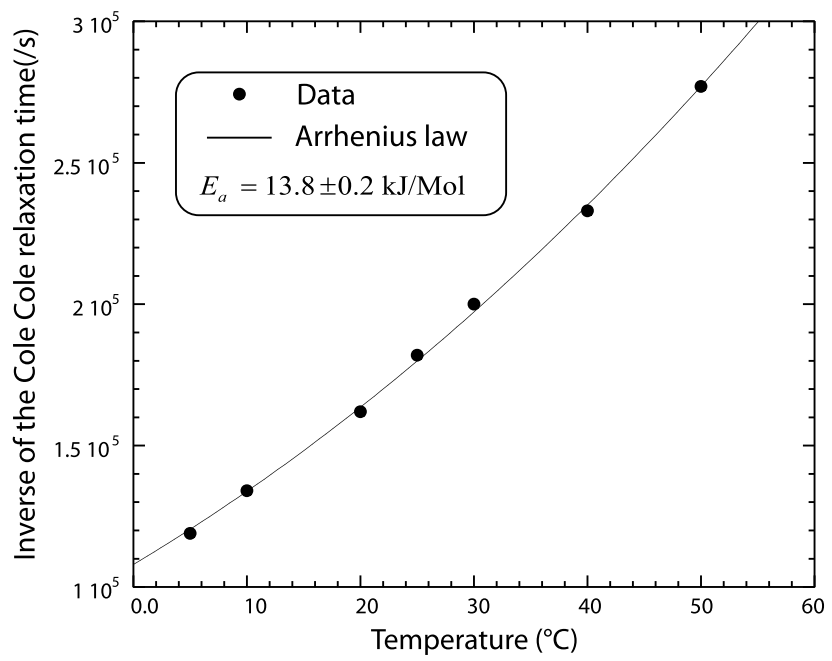

Figure 26. Dependence of the relaxation time with the temperature for the numerical simulations for a small grain of pyrite $(20 \mu \mathrm{m})$, for which the polarization is controlled by the formation of electricalfield-induced DLs. The fit is obtained with equation 17 . We obtain a relaxation time $\tau_{0}$ at $25^{\circ} \mathrm{C}$ of $(5.57 \pm 0.03) \times 10^{-6} \mathrm{~s} .\left(R^{2}=0.9989\right)$. We use a value of $15 \mathrm{~kJ} \mathrm{~mol}^{-1}$ for the activation energy of the ions for the background electrolyte.

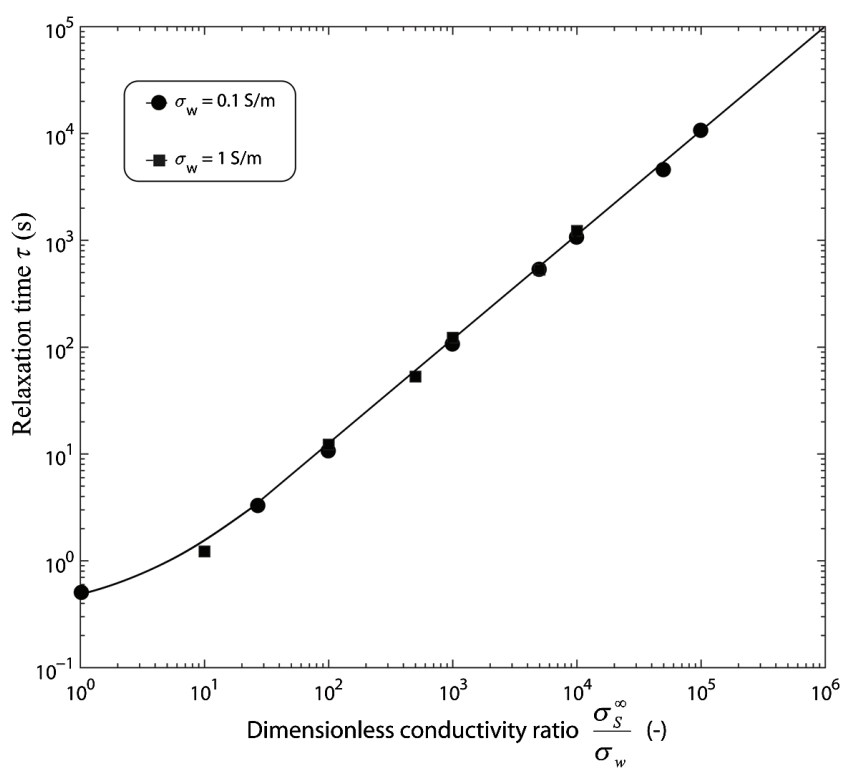

Figure 27. Finite-element simulations (filled symbols) of the polarization time constant $\tau$ associated with a single pyrite spherical grain (diameter $1 \mathrm{~cm}$ ) immersed in a background electrolyte of conductivity $\sigma_{w}$. We note that $\sigma_{S}^{\infty}$ is the instantaneous conductivity of the metallic grain. We observe that the relaxation time scales as $\left(1+\sigma_{S}^{\infty} / \sigma_{w}\right)$ (plain line). This model explains very well the observed dependence of the relaxation time with the salinity of the pore water. 
where $C_{m}$ denotes the concentration of the charge carriers in the solid metallic particle. For semiconductors, the dependence of the concentration of the charge carriers is temperature dependent and given approximately by

$$
C_{m}(T) \approx C_{m}^{0} T \exp \left(-\frac{\Delta E}{R T}\right) .
$$

This yields the following temperature dependence of the relaxation time:

$$
\tau_{i}(T)=\tau_{i}\left(T_{0}\right) \exp \left[\frac{E_{a}}{R}\left(\frac{1}{T}-\frac{1}{T_{0}}\right)\right],
$$

where the apparent activation energy is given by

$$
E_{a}=E_{a}^{w}-\Delta E
$$

This would lead to a reduction of the apparent activation energy with respect to the value in the pore water in agreement with observations. This calls for further future developments in the theory and numerical modeling that are too extensive to be reported here.

\section{CONCLUSION}

Spectral induced polarization measurements were performed in the temperature range $5^{\circ} \mathrm{C}-50^{\circ} \mathrm{C}$ and frequency range $10 \mathrm{mHz}$ to $45 \mathrm{kHz}$ for various metallic minerals (chalcopyrite, graphite, galena, magnetite, and pyrite) in a background clean sand that was independently characterized. For one pyrite cube, we investigated the complex conductivity spectra at six distinct salinities ( $\mathrm{NaCl}$ solutions). A 2D finiteelement model was used to understand the nature of the polarization mechanism for a small $(20 \mu \mathrm{m})$ pyrite grain immersed into a background 1:1 electrolyte. The following conclusions have been reached:

- The quadrature conductivity spectra show a clear peak that moves to higher frequencies when the temperature and/or salinity are increased. The shape of the spectra remains the same.

- A Cole-Cole model reproduces fairly well the data. The Cole-Cole parameters have a clear physical meaning from which their temperature dependence can be, in principle, inferred.

- The Cole-Cole exponent describes the broadness of the relaxation time distribution. When only one mechanism of polarization is at play (or dominates the overall response), all the relaxation times are expected to have the same temperature dependence, and therefore the Cole-Cole exponent is expected to be temperature independent. This is in agreement with the experimental data. The Cole-Cole exponent is also independent of the salinity in agreement with the experimental data.

- The chargeability is temperature and salinity independent and depends solely on the volumetric content of metallic particles and the chargeability of the background material.

- The instantaneous conductivity and steady-state conductivity depends linearly on temperature with a thermal sensitivity coefficient of $0.021 \pm 0.01^{\circ} \mathrm{C}^{-1}$ (equivalent to an activation energy of $14-16 \mathrm{~kJ} \mathrm{Mol}^{-1}$ ). This temperature coefficient (activation energy) is the same as describing the dynamic viscosity of the pore water. This is expected because the instantaneous conductivity and steady-state conductivity are related to the conductivity of the background (the material surrounding the metallic particles) and to the volumetric content of metallic particles. The conductivity of the material is in turn controlled by the conductivity of the pore water, and its temperature dependence is controlled by the viscosity of the pore water.

- The Cole-Cole relaxation time decreases with the increase of temperature. The mean value of the activation energy and standard deviations for the Cole-Cole relaxation time are $7.9 \pm 4.2 \mathrm{~kJ} \mathrm{Mol}^{-1}$; they are therefore smaller than for the DC and instantaneous conductivities and may depend on the mineral type. These values should be seen as indicative rather than as definitive.

- The previous results are confirmed by a 2D finite-element simulation in which we model the polarization of a semiconductor immersed in a background 1:1 electrolyte. In the numerical simulations, changing the activation energy of the charge carriers in the metallic particle has little influence on the apparent activation energy controlling the relaxation time. This is only true for very small metallic grains $(20 \mu \mathrm{m})$, whereas the laboratory experiments were performed with large metallic grains $(\gg 500 \mu \mathrm{m})$. The results indicate that the chargeability and the Cole-Cole exponent do not depend on the temperature and that the Cole-Cole relaxation time can be modeled with an Arrhenius law. However, we show that for small metallic grains $(<500 \mu \mathrm{m})$, the relaxation time can be controlled by the formation of field-induced electrical DLs, whereas for large metallic grains $(>500 \mu \mathrm{m})$, the relaxation time seems to be controlled by the relaxation of the charge carriers inside the metallic particles. For our numerical simulations with large metallic grains $(>500 \mu \mathrm{m})$, we also neglected the formation of DL induced polarization phenomena inside the bulk electrolyte. In this case, the simulations show that the relaxation time inversely depends on the conductivity of the background electrolyte in agreement with the experimental evidences obtained in this paper and by others.

- Our model also implies that the porosity of the background material affects the formation factor and therefore the instantaneous conductivity and DC conductivity of the mixture. In turn, the change of the background conductivity can affect the position of the peak frequency in agreement with finiteelement numerical simulations. In addition, different metallic minerals with the same grain size cannot be discriminated using spectral induced polarization data (at least in the linear regime). That said, in the field, different mineralizations (e.g., graphite in shale and sulfide deposits) are likely to be characterized by different grain sizes and therefore their polarizations are expected to occur in different frequency bands.

\section{ACKNOWLEDGMENTS}

We thank N. Florsch for the useful discussions. We also thank A. Weller, an anonymous referee, and the associate editor for fruitful comments that have helped us to improve the manuscript. 


\section{DATA AND MATERIALS AVAILABILITY}

Data associated with this research are available and can be obtained by contacting the corresponding author.

\section{APPENDIX A}

\section{ARRHENIUS ACTIVATION ENERGY}

We discuss here the temperature dependence of the diffusion coefficient of the ions in the electrolyte. Typically, the diffusion coefficient is related to the dynamic viscosity of the pore water $\eta_{w}$ (in $\mathrm{Pa} \mathrm{s}$ ) by the Stokes-Einstein relationship given by

$$
D=\frac{k_{b} T}{6 \pi r \eta_{w}},
$$

where $r$ denotes the radius of the diffusing species in the solvent (water) and is determined by the molecular volume of the species. The temperature dependence of the viscosity can be written according to the Vogel-Fulcher-Tammann equation (e.g., Vogel, 1921)

$$
\eta_{w}(T)=\eta_{\mathrm{o}} \exp \left(\frac{E_{a}^{w}}{R T}\right)
$$

in the context of the transition-state theory of Arrhenius chemical kinetics to transport phenomena. It follows that the temperature dependence of the ion diffusion coefficient is given by

$$
D(T)=\frac{k_{b} T}{6 \pi r \eta_{\mathrm{o}}} \exp \left(\frac{E_{a}^{w}}{R T}\right),
$$

or alternatively by

$$
D(T)=D\left(T_{0}\right) \frac{T}{T_{0}} \exp \left[-\frac{E_{a}^{w}}{R}\left(\frac{1}{T}-\frac{1}{T_{0}}\right)\right] .
$$

\section{APPENDIX B}

\section{PYRITE GRAIN IMMERSED IN AN ELECTROLYTE}

In this appendix, we discuss the key equations needed to numerically model the polarization around a grain of a semiconductor in a background electrolyte (see Wong, 1979; Wong and Strangway, 1981; Chu and Bazant, 2006; Gurin et al., 2015; Misra et al., 2016a, 2016b for further readings on this subject). The equations can be written either in the frequency or time domain. We consider an applied harmonic field such as $\mathbf{E}_{a}=\mathbf{E}_{0} \exp (i \omega t)$. The electrolyte around the pyrite grain is a binary symmetric $1: 1$ electrolyte such as $\mathrm{NaCl}$ or $\mathrm{KCl}$ without redox active species. The concentrations and electrical potential are given by the sum of values at equilibrium plus perturbations

$$
\begin{gathered}
C_{( \pm)}(\mathbf{r}, t)=C_{( \pm)}^{0}+\delta C_{( \pm)}(\mathbf{r}, t) e^{i \omega t}, \\
\psi_{e}(\mathbf{r}, t)=\delta \psi_{e}(\mathbf{r}, t) e^{i \omega t}, \\
C_{(+)}^{0}=C_{(-)}^{0},
\end{gathered}
$$

where $\psi_{e}$ describes the electrical potential that is external to the metallic particle and $C_{( \pm)}^{0}$ describes the equilibrium concentrations of the ions in the electrolyte in the absence of an external applied field.

The current density is controlled by the occurrence of a gradient in the electrochemical potentials of the charge carriers in the electrolyte, which can be expressed as

$$
\nabla \tilde{\mu}_{( \pm)}=-( \pm e) \mathbf{E}+k_{b} T \nabla \ln C_{( \pm)},
$$

where $e$ denotes the elementary charge $\left(1.6 \times 10^{-19} \mathrm{C}\right), k_{b}$ is the Boltzmann constant, and $T$ is the absolute temperature (in $\mathrm{K}$ ). The different mobilities can be defined by

$$
\beta_{( \pm)}=\left|q_{( \pm)}\right| b_{( \pm)}=\frac{\left|q_{( \pm)}\right| D_{( \pm)}}{k_{b} T},
$$

where $q_{( \pm)}$denotes the charge of the cations and anions. In the following, we express the problem in terms of the diffusion coefficient only, but equation B-5 can be used to rewrite the corresponding equations to alternative expressions explicitly using the mobilities rather than the diffusion coefficients. The diffusion flux densities can be written as

$$
\mathbf{J}_{( \pm)}=-b_{( \pm)} C_{( \pm)} \nabla \tilde{\mu}_{( \pm)},
$$

$$
\mathbf{J}_{( \pm)}=\left[( \pm e) \frac{D_{( \pm)} C_{( \pm)}^{0}}{k_{b} T} \nabla \delta \psi_{e}(\mathbf{r}, t)-D_{( \pm)} \nabla \delta C_{( \pm)}(\mathbf{r}, t)\right] e^{i \omega t}
$$

The continuity equation for the ionic species can be expressed as

$$
\nabla \cdot \mathbf{J}_{( \pm)}=-\frac{\partial C_{( \pm)}(\mathbf{r}, t)}{\partial t} .
$$

Combining equations B-7 and B-8 yields

$$
\begin{aligned}
& ( \pm e) \frac{D_{( \pm)} C_{( \pm)}^{0}}{k_{b} T} \nabla^{2} \delta \psi_{e}(\mathbf{r}, t)-D_{( \pm)} \nabla^{2} \delta C_{( \pm)}(\mathbf{r}, t) \\
& =-i \omega \delta C_{( \pm)}(\mathbf{r}, t) .
\end{aligned}
$$

The fluctuations in the electrical potential and the ionic concentrations are also given by a Poisson equation based on Gauss's law:

$$
\begin{gathered}
\varepsilon_{f} \nabla \cdot \mathbf{E}=\rho, \\
\rho=e\left[C_{(+)}(\mathbf{r}, t)-C_{(-)}(\mathbf{r}, t)\right] .
\end{gathered}
$$

This yields

$$
\nabla^{2} \delta \psi_{e}(\mathbf{r}, t)=-\frac{N e}{\varepsilon_{f}}\left[\delta C_{(+)}(\mathbf{r}, t)-\delta C_{(-)}(\mathbf{r}, t)\right] .
$$

The same type of equations can be written inside of the metallic particle for the two charge carriers: electrons and p-holes, which also obeys the electrochemical potential gradients: 


$$
\begin{aligned}
& ( \pm e) \frac{D_{( \pm)}^{m} C_{( \pm)}^{m}}{k_{b} T} \nabla^{2} \delta \psi_{i}(\mathbf{r}, t)-D_{( \pm)}^{m} \nabla^{2} \delta C_{( \pm)}^{m}(\mathbf{r}, t) \\
& =-i \omega \delta C_{( \pm)}^{m}(\mathbf{r}, t) \\
& \nabla^{2} \delta \psi_{i}(\mathbf{r}, t)=-\frac{N e}{\varepsilon_{S}}\left[\delta C_{(+)}^{m}(\mathbf{r}, t)-\delta C_{(-)}^{m}(\mathbf{r}, t)\right],
\end{aligned}
$$

where $D_{( \pm)}^{m}$ denotes the diffusion coefficient of the positive charges (p-holes) and electrons in the semiconductor, $\varepsilon_{S}$ denotes the dielectric constant of the solid grain, and $\delta \psi_{i}$ is the perturbed potential inside the metallic particle. To simulate the effect of temperature, we assume that the diffusion coefficient of the ions and the electrons/p-holes follow an Arrhenius type law; i.e.

$$
\begin{aligned}
& D_{( \pm)}(T)=D_{( \pm)}\left(T_{0}\right) \frac{T}{T_{0}} \exp \left[-\frac{E_{a}^{w}}{R}\left(\frac{1}{T}-\frac{1}{T_{0}}\right)\right], \\
& D_{( \pm)}^{m}(T)=D_{( \pm)}^{m}\left(T_{0}\right) \frac{T}{T_{0}} \exp \left[-\frac{E_{a}^{m}}{R}\left(\frac{1}{T}-\frac{1}{T_{0}}\right)\right] .
\end{aligned}
$$

The solution of the previous system of equations was calculated in the time domain (constant electrical potential during injection $T_{\text {on }}$ and null during $T_{\text {off }}$ ) using a Dirichlet boundary condition to the right and left sides of the domain. We also use the Neumann condition at the top and bottom of the model where we assume that no flux of charge is allowed (the normal derivative of the potential is null). This corresponds to insulating the boundary conditions at these frontiers. On the metallic grain, we impose a blocking boundary condition on the pore water/metallic grain interface. The charge concentration and charge mobility in each zone (grain and electrolyte) are defined using a space function, the ions are defined in the electrolyte, whereas electrons and holes are defined in the metallic grain (i.e., the holes and electron concentration are null outside the grain). At the beginning of calculation $(t=0)$, we assume the following initial conditions: (1) The potential is zero everywhere in the medium (no external electric field), (2) the electron and p-hole concentrations are equal and homogeneous in the semiconductor grain, and (3) the cation and anion concentrations are equal and homogeneous in the electrolyte.

For large metallic grains $(>500 \mu \mathrm{m}$, see the discussion in the main text), we neglect the diffusion of the charge carriers in the surrounding electrolyte, i.e., we consider that the formation of induced DL does not control the overall polarization process. This is done by putting the concentration gradient term equal to zero in the NernstPlanck equation in the electrolyte.

\section{REFERENCES}

Abdel Aal, G. Z., E. A. Atekwana, and A. Revil, 2014, Geophysical signatures of disseminated iron minerals: A proxy for understanding subsurface biophysicochemical processes: Journal of Geophysical Research, 119, 1831-1849.

Abdulsamad, F., N. Florsch, and C. Camerlynck, 2017, Spectral induced polarization in a sandy medium containing semiconductor materials: Experimental results and numerical modeling of the polarization mechanism: Near Surface Geophysics, 15, 669-683.

Bairlein, K., M. Bücker, A. Hördt, and B. Hinze, 2016, Temperature dependence of spectral induced polarization data: Experimental results and mem- brane polarization theory: Geophysical Journal International, 205, 440453, doi: $10.1093 /$ gji/ggw027.

Binley, A., S. Kruschwitz, D. Lesmes, and N. Kettridge, 2010, Exploiting the temperature effects on low frequency electrical spectra of sandstone: A comparison of effective diffusion path lengths: Geophysics, 75, no. 6, A43-A46, doi: 10.1190/1.3483815.

Campbell, R. B., C. A. Bower, and L. A. Richards, 1948, Change in electrical conductivity with temperature and the relation of osmotic pressure to electrical conductivity and ion concentration for soil extracts: Soil Science Society of America Proceedings, 13, 66-69, doi: 10.2136/sssaj1949 $.036159950013000 \mathrm{C} 0010 \mathrm{x}$.

Chen, J., A. Kemna, and S. S. Hubbard, 2008, A comparison between GaussNewton and Markov-chain Monte Carlo-based methods for inverting spectral induced-polarization data for Cole-Cole parameters: Geophysics, 73, no. 6, F247-F259, doi: 10.1190/1.2976115.

Chu, K. T., and M. Z. Bazant, 2006, Nonlinear electrochemical relaxation around conductors: Physical Review E, 74, 011501, doi: 10.1103/ PhysRevE.74.011501.

Flekkøy, E. G., 2013, A physical basis for the Cole-Cole description of electrical conductivity of mineralized porous media: Geophysics, 78 , no. 5 , D353-D366, doi: https://doi.org/10.1190/geo2012-0478.1.

Flores Orozco, A., K. H. Williams, P. E. Long, S. S. Hubbard, and A. Kemna, 2011, Using complex resistivity imaging to infer biogeochemical processes associated with bioremediation of an uranium-contaminated aquifer: Journal of Geophysical Research, 116, G03001.

Florsch, N., C. Camerlynck, and A. Revil, 2012, Direct estimation of the distribution of relaxation times from induced-polarization spectra using a Fourier transform analysis: Near Surface Geophysics, 10, 517-531.

Frische, R. H., and H. von Buttlar, 1957, A theoretical study of induced electrical polarization: Geophysics, 22, 688-706, doi: 10.1190/1.1438404.

Ghorbani, A., C. Camerlynck, N. Florsch, P. Cosenza, A. Tabbagh, and A. Revil, 2007, Bayesian inference of the Cole-Cole parameters from time and frequency-domain induced polarization: Geophysical Prospecting, 55, 589-605, doi: 10.1111/j.1365-2478.2007.00627.x.

Gurin, G., K. Titov, Y. Ilyin, and A. Tarasov, 2015, Induced polarization of disseminated electronically conductive minerals: A semi-empirical model: Geophysical Journal International, 200, 1555-1565, doi: 10.1093/gji/ ggu490.

Hupfer, S., T. Martin, A. Weller, T. Günther, K. Kuhn, V. Djotsa Nguimeya Ngninjio, and U. Noell, 2016, Polarization effects of unconsolidated sulphide-sand-mixtures: Journal of Applied Geophysics, 135, 456-465, doi: 10.1016/j.jappgeo.2015.12.003.

Kemna, A., A. Binley, G. Cassiani, E. Niederleithinger, A. Revil, L. Slater, K. H. Williams, A. Flores Orozco, F.-H. Haegel, A. Hördt, S. Kruschwitz, V. Leroux, K. Titov, and E. Zimmermann, 2012, An overview of the spectral induced polarization method for near-surface applications: Near Surface Geophysics, 10, 453-468.

Liu, W., R. Chen, H. Cai, W. Luo, and A. Revil, 2017, Correlation analysis for spread spectrum induced polarization signal processing in electromagnetically noisy environments: Geophysics, 82, no. 5, E243-E256, doi: 10 .1190/GEO2016-0109.1.

Mahan, M. K., J. D. Redman, and D. W. Strangway, 1986, Complex resistivity of synthetic sulfide bearing rocks: Geophysical Prospecting, 34 743-768, doi: 10.1111/j.1365-2478.1986.tb00491.x.

Mao, D., and A. Revil, 2016, Induced polarization response of porous media with metallic particles. Part 3: A new approach to time-domain induced polarization tomography: Geophysics, 81, no. 4, D345-D357, doi: 10 .1190/geo2015-0283.1.

Mao, D., A. Revil, and J. Hinton, 2016, Induced polarization response of porous media with metallic particles. Part 4: Detection of metallic and non-metallic targets in time domain induced polarization tomography: Geophysics, 81, no. 4, D359-D375, doi: 10.1190/geo2015-0480.1.

Meju, M., 2002, Geoelectromagnetic exploration for natural resources: Models, case studies and challenges: Surveys in Geophysics, 23, 133 206, doi: 10.1023/A:1015052419222.

Mewafy, F. M., D. D. Werkema, E. A. Atekwana, L. D. Slater, G. Z. Abdel Aal, A. Revil, and D. Ntarlagiannis, 2013, Evidence that bio-metallic mineral precipitation enhances the complex conductivity response at a hydrocarbon contaminated site: Journal of Applied Geophysics, 98, 113-123, doi: 10.1016/j.jappgeo.2013.08.011.

Misra, S., C. Torres-Verdín, A. Revil, J. Rasmus, and D. Homan, 2016a, Interfacial polarization of disseminated conductive minerals in absence of redox-active species. Part 1: Mechanistic model and validation: Geophysics, 81, no. 2, E139-E157, doi: 10.1190/geo2015-0346.1.

Misra, S., C. Torres-Verdín, A. Revil, J. Rasmus, and D. Homan, 2016b, Interfacial polarization of disseminated conductive minerals in absence of redox-active species. Part 2: Effective complex conductivity and dielectric permittivity: Geophysics, 81, no. 2, E159-E176, doi: 10.1190/ geo2015-0400.1.

Ntarlagiannis, D., K. H. Williams, L. Slater, and S. Hubbard, 2005, Low frequency electrical response to microbial induced sulfide precipitation: Journal of Geophysical Research, 110, G02009. 
Olhoeft, G. R., 1985, Low-frequency electrical properties: Geophysics, 50, 2492-2503, doi: $10.1190 / 1.1441880$.

Ostrander, A. G., and K. L. Zonge, 1978, Complex resistivity measurements of sulfide-bearing synthetic rocks: 48th Annual International Meeting, SEG, Expanded Abstracts, M-6, 113 .

Pelton, W. H., S. H. Ward, P. G. Hallof, W. R. Sill, and P. H. Nelson, 1978, Mineral discrimination and removal of inductive coupling with multifrequency IP: Geophysics, 43, 588-609, doi: 10.1190/1.1440839.

Phillips, C. R., 2010, Experimental study of the induced polarization effect using Cole-Cole and GEMTIP models: M.S. thesis, University of Utah.

Rajeshwar, K., 2007, Fundamentals of semiconductor electrochemistry and photoelectrochemistry: Wiley-VCH Verlag $\mathrm{GmbH} \& \mathrm{Co}$.

Revil, A., G. Z. Abdel Aal, E. A. Atekwana, D. Mao, and N. Florsch, 2015b, Induced polarization response of porous media with metallic particles. Part 2: Comparison with a broad database of experimental data: Geophysics, 80, no. 5, D539-D552, doi: 10.1190/geo2014-0578.1.

Revil, A., A. Coperey, Z. Shao, N. Florsch, I. L. Fabricius, Y. Deng, J. R. Delsman, P. S. Pauw, M. Karaoulis, P. G. B. de Louw, E. S. van Baaren, W. Dabekaussen, A. Menkovic, and J. L. Gunnink, 2017c, Complex conductivity of soils: Water Resources Research, 53, 7121-7147, doi: 10 .1002/2017WR020655.

Revil, A., N. Florsch, and D. Mao, 2015a, Induced polarization response of porous media with metallic particles. Part 1: A theory for disseminated semiconductors: Geophysics, 80, no. 5, D525-D538, doi: 10.1190/ geo2014-0577.1

Revil, A., M. Karaoulis, T. Johnson, and A. Kemna, 2012, Review: Some low-frequency electrical methods for subsurface characterization and monitoring in hydrogeology: Hydrogeology Journal, 20, 617-658, doi: 10.1007/s10040-011-0819-X

Revil, A., D. Mao, Z. Shao, M.F. Sleevi, and D. Wang, 2017b, Induced polarization response of porous media with metallic particles. Part 6: The case of metals and semi-metals: Geophysics, 82, no. 2, E97-E110, doi: 10 $.1190 /$ geo2016-0389.1

Revil, A., and M. Skold, 2011, Salinity dependence of spectral induced polarization in sands and sandstones: Geophysical Journal International, 187, 813-824, doi: 10.1111/j.1365-246X.2011.05181.x.

Revil, A., M. F. Sleevi, and D. Mao, 2017a, Induced polarization response of porous media with metallic particles. Part 5: Influence of the background polarization: Geophysics, 82, no. 2, E77-E96, doi: 10.1190/geo20160388.1 .

Schlumberger, C., 1920, Etude sur la prospection électrique du sous-sol: Gauthier-Villars.

Seigel, H. O., 1959, Mathematical formulation and type curves for induced polarization: Geophysics, 24, 547-565, doi: 10.1190/1.1438625.

Shen, P., Y. Sheng, and T. Liu, 2007, Application of stratagem EH4 system to prediction of hidden ore bodies: Mineral Deposits, 26, 70-78.

Slater, L., J. Choi, and Y. Wu, 2005, Electrical properties of iron-sand columns: Implications for induced polarization investigations and performance monitoring of iron-wall barriers: Geophysics, 70, no. 4, G87-G94, doi: 10.1190/1.1990218.

Tarasov, A., and K. Titov, 2013, On the use of the Cole-Cole equations in spectral induced polarization: Geophysical Journal International, 195, 352-356, doi: $10.1093 /$ gji/ggt251.
Tombs, J. M. C., 1981, The feasibility of making spectral IP measurements in the time domain: Geoexploration, 19, 91-102, doi: 10.1016/0016-7142 (81)90022-3

Tong, M., and H. Tao, 2007, Experimental study of induced polarization relaxation time spectra of shaly sands: Journal of Petroleum Science and Engineering, 59, 239-249, doi: 10.1016/j.petrol.2007.04.004.

Toumi, A., N. Hafaiedh, and M. Bouanz, 2009, Thermodynamic properties of triethylamine + water liquid mixture from shear viscosity measurements: Fluid Phase Equilibria, 278, 68-75, doi: 10.1016/j.fluid.2009 .01 .004 .

Vacquier, V., C. R. Holmes, P. R. Kintzinger, and M. Lavergne, 1957, Prospecting for ground water by induced electrical polarization: Geophysics, 22, 660-687, doi: 10.1190/1.1438402.

Veeken, P, P. Legeydo, Y. Davidenko, E. Kudryavceva, S. Ivanov, and A. Chuvaev, 2009, Benefits of the induced polarization geo-electric method to hydrocarbon exploration: Geophysics, $\mathbf{7 4}$, no. 2, B47-B59, doi: 10 $1190 / 1.3076607$

Viktor, P. J., and D. K. Hazra, 2002, Excess molar volumes, viscosity deviations, and isentropic compressibility changes in binary mixtures of $\mathrm{N}$-methylacetamide +2 -methoxyethanol and Nmethylacetamide + water at $(308.15,313.15$, and 318.15$) \mathrm{K}$ : Journal of Chemical \& Engineering Data, 47, 79-82.

Vinegar, H., and M. Waxman, 1984, Induced polarization of shaly sands: Geophysics, 49, 1267-1287, doi: 10.1190/1.1441755.

Vogel, H., 1921, Das Temperaturabhangigkeitsgesetz der Viskositat von Flussigkeiten: Physikalische Zeitschrift, 22, 645-646.

Wait, J. R., 1959, Overvoltage research and geophysical applications: Pergamon.

Wong, J., 1979, An electrochemical model of the induced-polarization phenomenon in disseminated sulfide ores: Geophysics, 44, 1245-1265, doi: $10.1190 / 1.1441005$.

Wong, J., and D. Strangway, 1981, Induced polarization in disseminated sulfide ores containing elongated mineralization: Geophysics, 46, 1258-1268, doi: 10.1190/1.1441264.

Woodruff, W. F., A. Revil, and C. Torres-Verdín, 2014, Laboratory determination of the complex conductivity tensor of unconventional anisotropic shales: Geophysics, 79, no. 5, E183-E200, doi: 10.1190/geo2013-0367.1.

Yoshioka, K., and M. S. Zhdanov, 2005, Three-dimensional non-linear regularized inversion of the induced polarization data based on the Cole-Cole model: Physics of the Earth and Planetary Interiors, 150, 29-43.

Zimmermann, E., J. Berwix, W. Glaas, H. Meier, H. M. Münch, and A. Kemna, 2007, ZEL-SIP04-V02: User manual: Forschungszentrum Julich $\mathrm{GmbH}$.

Zisser, N., A. Kemna, and G. Nover, 2010, Dependence of spectral induced polarization response of sandstone on temperature and its relevance to permeability estimation: Journal of Geophysical Research, 115, doi: 10 $.1029 / 2010 J B 007526$.

Zonge, K., and J. Wynn, 1975, Recent advances and applications in complex resistivity measurements: Geophysics, 40, 851-864, doi: 10.1190/1.1440572.

Zonge, K. L., and L. J. Hughes, 1981, The complex resistivity method, in Advances in induced polarization and complex resistivity (short course): 5-7 January 1981, Laboratory of Geophysics: University of Arizona Press, 163-208. 